\title{
Rare event asymptotics for a random walk in the quarter plane
}

\author{
Fabrice Guillemin • Johan S.H. van Leeuwaarden
}

Received: 8 August 2009 / Revised: 21 September 2010 / Published online: 11 November 2010

(C) The Author(s) 2010. This article is published with open access at Springerlink.com

\begin{abstract}
This paper presents a novel technique for deriving asymptotic expressions for the occurrence of rare events for a random walk in the quarter plane. In particular, we study a tandem queue with Poisson arrivals, exponential service times and coupled processors. The service rate for one queue is only a fraction of the global service rate when the other queue is non-empty; when one queue is empty, the other queue has full service rate. The bivariate generating function of the queue lengths gives rise to a functional equation. In order to derive asymptotic expressions for large queue lengths, we combine the kernel method for functional equations with boundary value problems and singularity analysis.
\end{abstract}

Keywords Boundary value problems $\cdot$ Random walks in the quarter plane $\cdot$ Rare events · Queueing theory · Singularity analysis · Tail decay rate · Large deviations

Mathematics Subject Classification (2000) 60K25 · 60F10

\section{Introduction}

Stationary distributions of two-dimensional one-step random walks in the quarter plane can be obtained by solving functional equations. Malyshev pioneered this general problem in the 1970's, and the theory has advanced since via its use in applications like lattice path counting and two-server queueing models. The idea of re-

F. Guillemin

Orange Labs, 2 Avenue Pierre Marzin, 22300 Lannion, France

e-mail: fabrice.guillemin@ orange-ftgroup.com

J.S.H. van Leeuwaarden ( $₫)$

Department of Mathematics and Computer Science, Eindhoven University of Technology,

P.O. Box 513, 5600 MB Eindhoven, The Netherlands

e-mail: j.s.h.v.leeuwaarden@tue.nl 
ducing the functional equation for the generating function to a standard RiemannHilbert boundary value problem stems from the work of Fayolle and Iasnogorodski [10] on two parallel $M / M / 1$ queues with coupled processors (the service speed of a server depends on whether or not the other server is busy). Extensive treatments of the boundary value technique for functional equations can be found in Cohen and Boxma [5] and Fayolle, Iasnogorodski and Malyshev [11]. This technique concerns sophisticated complex analysis, Riemann surfaces and various boundary value problems.

This paper presents a novel technique for deriving asymptotic estimates for the occurrence of certain types of rare events in a random walk in the quarter plane. We believe that our technique might prove useful in the analysis of a wider class of random walks, but in this paper we concentrate on a tandem queue with Poisson arrivals, exponential service times and coupled processors. Denote by $N_{1}$ and $N_{2}$ the stationary number of customers in the first and second queue. The generating function $P(x, y)=\mathbb{E}\left(x^{N_{1}} y^{N_{2}}\right)$ then satisfies the functional equation

$$
h_{1}(x, y) P(x, y)=h_{2}(x, y) P(x, 0)+h_{3}(x, y) P(0, y)+h_{4}(x, y) P(0,0) \text {, }
$$

where the functions $h_{j}$ are quadratic polynomials in $x$ and $y$. Equation (1) cannot be solved directly for $P(x, y)$, because it contains other unknown functions $P(x, 0)$ and $P(0, y)$. The classical approach is then to consider the roots of the kernel $h_{1}(x, y)$ w.r.t. one of the variables $x, y$. Substituting such roots into (1) yields additional relations between the unknown functions $P(x, 0)$ and $P(0, y)$. These relations give rise to boundary value problems whose solutions lead to a specification of $P(x, 0)$ and $P(0, y)$ and hence $P(x, y)$. For the tandem queue with coupled processors this was done in $[28,32]$. The formal solution obtained, however, is too complicated to invert for the stationary distribution. We shall look at this inversion problem, and derive asymptotic expressions for the marginal distributions.

To analyze $\mathbb{P}\left(N_{1}=n\right)$, for large $n$, we need to extract information from the generating function $P(x, 1)=\sum_{n=0}^{\infty} \mathbb{P}\left(N_{1}=n\right) x^{n}$. We shall employ the functional equation to determine the dominant (closest to the origin) singularities of the functions $P(x, 0)$ and $P(x, 1)$. Let $\xi$ denote the dominant singularity of $P(x, 1)$. Large deviations theory typically focusses on rough tail asymptotics of the form (the pole $\xi$ is positive)

$$
\lim _{n \rightarrow \infty} \frac{1}{n} \log \mathbb{P}\left(N_{1}=n\right)=-\log \xi
$$

We shall derive the exact tail asymptotics, which requires the investigation of $P(x, 1)$ in the neighborhood of its dominant singularity $\xi$.

\subsection{Singularity analysis}

In $[28,32]$ solutions for $P(x, 0)$ and $P(0, y)$ were derived that are valid only in certain parts of the complex planes. In this paper we provide complete solutions to $P(x, 0)$ and $P(0, y)$ that are in fact the analytic continuations to the entire complex planes of the solutions in $[28,32]$. The technique of investigating a function near its dominant singularity to obtain asymptotic expressions for its coefficients is known 
as singularity analysis and has a long history in areas of mathematics like analysis, combinatorics and number theory; for an elaborate exposition see Flajolet and Sedgewick [12]. In most cases the generating function is univariate and explicit, and extracting information on the coefficients boils down to the (asymptotic) evaluation of univariate contour integrals. The work on obtaining asymptotics from multivariate generating functions has been strongly motivated by recursively defined combinatorial structures like trees, see e.g. [9, 12, 13], and specific random walks or queueing models $[4,14,15,19]$. One of the central ideas in multivariate asymptotics is to exploit a functional equation to reduce multivariate problems to univariate contour integrals. In contrast to most functional equations that are subject to multivariate asymptotics (see [27] for an overview), our functional equation (1) does not allow for a closed-form solution, which complicates considerably the application of singularity analysis. Our method can be considered as a contribution to the technique of singularity analysis for bivariate generating functions.

Related work was done in [19] for two parallel $M / M / 1$ queues with coupled processors, also leading to rare event probabilities. However, this latter model can be reduced to a Dirichlet problem (the boundary value problem has a boundary which is a circle, and the problem is solved by using the Poisson kernel; see [10, 19]). In the present paper, the boundary is a general smooth closed contour and we use a Riemann-Hilbert formulation, which allows us to directly extend the function outside the domain delineated by the boundary. In this respect, the problem considered in the present paper is more general than the one considered in [19], and the approach that we take might prove useful for many models that fall into the class of random walks in the quarter plane.

\subsection{Alternative methods}

There are at least two alternative techniques to derive tail asymptotics for random walks in the quarter plane, perhaps the one with the longest tradition being large deviations theory. Seminal work in this area was done by Borovkov and Mogul'skii [2]. For so-called 0-partially homogeneous chains, of which our random walk in the quarter plane is a special case, in [2] (see also [21]) both the rough and the exact asymptotics have been considered. However, the decay rate is not made explicit, and it is not clear how to obtain results for marginal distributions. For determining the rough decay rate one needs to identify the local rate function and solve a variational problem. This variational problem, under additional assumptions, can be reduced to an optimization problem (see e.g. [30]), but requires a case-specific approach.

For the large deviations approach, it is necessary to incorporate the boundary effects, which requires finding an optimal path that minimizes a cost function. For many models, this gives rise to multiple regimes, where each regime corresponds to a certain most likely path. For a modified Jackson network, in which one server may help the other server, Foley and McDonald [17] were able to find most of these regimes using the large deviations approach. See also [1, 16, 18].

The second alternative techniques is the matrix-analytic method. Initially, the matrix-geometric method targeted at deriving the so-called boundary condition, under which the asymptotics show geometric behavior; see [20, 25, 31]. This boundary 
condition plays a crucial role in the large deviations approach too, and is naturally the subject of much recent work [20, 22, 23, 25, 29]. Geometric decay requires the dominant singularity to be a pole, whereas it could be a singularity of a different nature like a branch point. The recent work of Miyazawa [24] greatly enlarges the scope of applicability of the matrix-analytic techniques, because it is no longer restricted to the boundary condition. For the general class of skip-free random walks in the quarter plane, Miyazawa characterizes both the rough and exact asymptotics of the tail decay rates, for coordinate directions and marginal distributions. Among other things, it is shown in [24] that the matrix-analytic methods can be used to determine all asymptotic regimes for the modified Jackson network in [17].

\subsection{Contributions and outline of the paper}

The tandem queue with coupled processors, which we chose as our vehicle to present the asymptotic technique, is of independent interest. It arises as a natural model for bandwidth sharing of Internet capacity that is based on reservation procedures (see $[7,28,32])$. The two processors are coupled such that the speed of processor $i$ is $\mu_{i}$ when the other processor is busy, and $\mu_{i}^{*}$ when the other processor is idle. This coupling became extremely popular in the last decade due to its relation to the Generalized Processor Sharing (GPS) discipline $\left(\mu_{1}^{*}=\mu_{2}^{*}=\mu_{1}+\mu_{2}\right)$, the prevalent discipline for bandwidth sharing in packet networks. See [3] for an overview on GPS. The different asymptotic regimes identified in this paper yield structural insights on the impact of GPS on rare events in a tandem queue.

In the present paper, we make the following contributions:

- We provide in Propositions 3 and 4 exact solutions to $P(x, 0)$ and $P(0, y)$, in terms of meromorphic functions, which are valid in the entire complex $x$ and $y$ planes cut along some segments. The solutions follow from analytic continuations through the functional equation (1).

- We determine the domain of analyticity of the functions $P(x, 1)$ and $P(1, y)$. A crucial role is fulfilled by the resultant of the functions $h_{1}$ and $h_{2}$. The domains of analyticity lead to exact asymptotic expressions for $\mathbb{P}\left(N_{1}=n\right)$ and $\mathbb{P}\left(N_{2}=n\right)$.

- The parameter values determine the nature of the dominant singularities of $P(x, 1)$ and $P(1, y)$. This gives rise to several different asymptotic regimes. Asymptotic estimates for the probabilities of large queue lengths are obtained using Laplace's method and Darboux's method. Proposition 5 distinguishes four different regimes for queue 1, and Proposition 6 shows that there are three different regimes for queue 2 .

Section 2 contains the model description and an extensive analysis of the zero-pairs of the kernel $h_{1}$ in (1). In particular, various analytic continuations of these zero-pairs are constructed, which identify some of the singularities of the function $P(x, 0)$ and $P(0, y)$. Further singularities are identified in Sect. 3 by considering the resultant of $h_{1}$ and $h_{2}$. In Sect. 4 we formulate $P(x, 0)$ and $P(0, y)$ in terms of boundary value problems. The solutions to these boundary value problems yield solutions to $P(x, 0)$ and $P(0, y)$ in terms of meromorphic functions, with a clear description of their singularities. In Sect. 5 this knowledge is used to obtain a complete characterization of the exact asymptotics for the marginal distributions $\mathbb{P}\left(N_{1}=n\right)$ and $\mathbb{P}\left(N_{2}=n\right)$. 


\section{Model description and preliminary properties}

Consider a two-stage tandem queue, where jobs arrive at queue 1 according to a Poisson process with rate $\lambda$, demanding service at both queues before leaving the system. Each job requires an exponential amount of work with parameter $v_{j}$ at queue $j$. The global service rate is set to one. The service rate for one queue is only a fraction ( $p$ for queue 1 and $1-p$ for queue 2) of the global service rate when the other queue is non-empty; when one queue is empty, the other queue has full service rate. Therefore, when both queues are non-empty, the departure rates at queue 1 and 2 are $v_{1} p$ and $v_{2}(1-p)$, respectively.

When one of the queues is empty, the departure rate of the nonempty queue $j$ is temporarily increased to $v_{j}$. With $N_{j}(t)$ the number of jobs in queue $j$ at time $t$, the two-dimensional process $\left\{\left(N_{1}(t), N_{2}(t)\right), t \geq 0\right\}$ is a Markov process, and upon uniformization, a random walk in the quarter plane.

The ergodicity condition under which this Markov process has a unique stationary distribution is given by

$$
\rho=\frac{\lambda}{v_{1}}+\frac{\lambda}{v_{2}}<1
$$

This can be explained by the fact that, independent of $p$, the two stations together always work at capacity 1 (if there is work in the system), and that $\lambda / \nu_{1}+\lambda / \nu_{2}$ equals the amount of work brought into the system per time unit. We henceforth assume that the ergodicity condition is satisfied.

Denote the joint stationary probabilities by

$$
\mathbb{P}\left(N_{1}=n, N_{2}=k\right)=\lim _{t \rightarrow \infty} \mathbb{P}\left(N_{1}(t)=n, N_{2}(t)=k\right)
$$

and let $P(x, y)$ represent the bivariate generating function

$$
P(x, y)=\sum_{n=0}^{\infty} \sum_{k=0}^{\infty} \mathbb{P}\left(N_{1}=n, N_{2}=k\right) x^{n} y^{k} .
$$

From the balance equations it follows (see [28]) that $P(x, y)$ satisfies the functional equation (1) with

$$
\begin{aligned}
& h_{1}(x, y)=\left(\lambda+p v_{1}+(1-p) v_{2}\right) x y-\lambda x^{2} y-p v_{1} y^{2}-(1-p) v_{2} x, \\
& h_{2}(x, y)=(1-p)\left(v_{1} y(y-x)+v_{2} x(y-1)\right), \\
& h_{3}(x, y)=-\frac{p}{1-p} h_{2}(x, y), \\
& h_{4}(x, y)=v_{2} x(y-1)-h_{2}(x, y) .
\end{aligned}
$$

We have $P(0,0)=1-\rho$ by (2). 


\subsection{Zero-pairs of the kernel}

Let $\hat{r}=1+1 / r_{1}+1 / r_{2}$ with $r_{1}=\lambda /\left(p v_{1}\right)$ and $r_{2}=\lambda /\left((1-p) v_{2}\right)$. With this notation, the equation $h_{1}(x, y)=0$ in $y$ has two roots:

$$
X_{ \pm}(y)=\frac{1}{2 y}\left(\left(\hat{r} y-1 / r_{2}\right) \pm \sqrt{D_{2}(y)}\right)
$$

where

$$
D_{2}(y)=\left(\hat{r} y-1 / r_{2}\right)^{2}-4 y^{3} / r_{1} \text {. }
$$

The functions $X_{ \pm}(y)$ are well defined for $y \in \mathbb{R} \backslash\{0\}$ as long as $D_{2}(y) \geq 0$. It is easily checked that $\lim _{y \rightarrow 0} X_{+}(y)=0$ (the point 0 is a removable singularity for the function $\left.X_{+}(y)\right)$ and $\lim _{y \rightarrow 0} X_{-}(y)=-\infty$ (the point 0 is a singularity for the function $\left.X_{-}(y)\right)$. In addition, as shown in [32], the discriminant $D_{2}(y)$ has three roots in $\mathbb{R}$. These three roots are denoted by $y_{1}, y_{2}$ and $y_{3}$ and are such that $0<y_{1}<$ $y_{2} \leq 1<y_{3}$. We have $D_{2}(y)>0$ for $y \in\left(-\infty, y_{1}\right) \cup\left(y_{2}, y_{3}\right)$ and $D_{2}(y)<0$ for $y \in\left(y_{1}, y_{2}\right) \cup\left(y_{3}, \infty\right)$.

Similarly, the equation $h_{1}(x, y)=0$ in $x$ has two roots:

$$
Y_{ \pm}(x)=\frac{r_{1}}{2}\left((\hat{r}-x) x \pm \sqrt{D_{1}(x)}\right)
$$

where

$$
D_{1}(x)=((\hat{r}-x) x)^{2}-4 x /\left(r_{1} r_{2}\right) .
$$

The functions $Y_{ \pm}(x)$ are well defined for $x \in \mathbb{R}$ as long as the discriminant $D_{1}(x) \geq 0$. As shown in [32], the discriminant $D_{1}(x)$ has four real roots $x_{1}=0<$ $x_{2} \leq 1<x_{3}<x_{4}$. We have $D_{1}(x)>0$ for $x \in\left(-\infty, x_{1}\right) \cup\left(x_{2}, x_{3}\right) \cup\left(x_{4}, \infty\right)$ and $D_{1}(x)<0$ for $x \in\left(x_{1}, x_{2}\right) \cup\left(x_{3}, x_{4}\right)$.

In the next section we investigate how to analytically continue the functions $Y_{ \pm}(x)$ in $\mathbb{C} \backslash\left(\left[x_{1}, x_{2}\right] \cup\left[x_{3}, x_{4}\right]\right)$ and $X_{ \pm}(y)$ in $\mathbb{C} \backslash\left(\left[y_{1}, y_{2}\right] \cup\left[y_{3}, \infty\right)\right)$.

\subsection{Analytic continuation}

In the following, we assume that for $z \in \mathbb{C}, \arg (z) \in(-\pi, \pi]$, and we take the determination of the square root such that $\sqrt{x^{2}}=x$ if $x \geq 0$ and $\sqrt{-1}=i$. The couple $\left(X_{+}(y),\left(-\infty, y_{1}\right)\right)$ defines a germ of analytic function. We first investigate how this germ can be analytically continued in the complex plane deprived of the segments $\left[y_{1}, y_{2}\right]$ and $\left[y_{3}, \infty\right)$. Let $z^{+}=\Re(z)+i|\Im(z)|$.

\section{Lemma 1 The function}

$$
X^{*}(y)= \begin{cases}X_{+}(y) & \text { when } y \in\left\{z: \Re(z) \leq y_{2}, \Im\left(D_{2}\left(z^{+}\right)\right)<0\right\} \cup\left(-\infty, y_{1}\right), \\ X_{-}(y) \quad \text { otherwise }\end{cases}
$$

defined in $\mathbb{C} \backslash\left(\left[y_{1}, y_{2}\right] \cup\left[y_{3}, \infty\right)\right)$, is analytic. 
Proof Let $y=u+i v$ with $u, v \in \mathbb{R}$. We have $D_{2}(y)=\Re\left(D_{2}(y)\right)+i \Im\left(D_{2}(y)\right)$ with

$$
\begin{aligned}
& \Re\left(D_{2}(y)\right)=\left(\hat{r} u-\frac{1}{r_{2}}\right)^{2}-\hat{r}^{2} v^{2}-\frac{4}{r_{1}}\left(u^{3}-3 u v^{2}\right), \\
& \Im\left(D_{2}(y)\right)=v\left(\frac{4}{r_{1}} v^{2}-\left(\frac{12}{r_{1}} u^{2}-2 \hat{r}^{2} u+\frac{2}{r_{2}}\right)\right) .
\end{aligned}
$$

The imaginary part vanishes for $u$ and $v$ satisfying the equation

$$
\frac{4}{r_{1}} v^{2}=\frac{12}{r_{1}} u^{2}-2 \hat{r}^{2} u+\frac{2}{r_{2}} .
$$

For sufficiently large $u$, the right-hand side of the above equation is positive. If we assume that this term does not cancel for $u$ describing the whole of $\mathbb{R}$, then we can define two curves in $\mathbb{C}$ along which the imaginary part of $D_{2}(y)$ vanishes: one curve lies entirely in the positive half-plane $\{y: \Im(y)>0\}$ and the other curve lies entirely in the negative half-plane $\{y: \Im(y)<0\}$.

Along one of these curves, the sign of the real part $\Re\left(D_{2}(y)\right)$ is constant since the imaginary and real parts cancel only for $y \in \mathbb{R}$ (namely for $y$ equal to one of the roots $y_{1}, y_{2}$ and $y_{3}$ ). For the curve in the upper half-plane we have $v^{2} \sim 3 u^{2}$ for $|u| \rightarrow+\infty$. But in this case, we would have $\mathfrak{R}\left(D_{2}(y)\right) \sim 32 u^{3} / r_{1}$, which contradicts the fact that $\Re\left(D_{2}(y)\right)$ should keep the same sign as $u$ describes the whole of $\mathbb{R}$. Hence, the polynomial in the right-hand side of (6) has roots in $\mathbb{R}$, which are positive since the value of this polynomial at 0 is $2 / r_{2}>0$. Let $y_{1}^{*}$ and $y_{2}^{*}$ denote these roots with $0<y_{1}^{*} \leq y_{2}^{*}$.

Equation (6) defines two hyperbolic branches as depicted in Fig. 1. The left branch intersects the real axis at point $y_{1}^{*}$ and for a point $y$ on this branch such that $\Im(y) \neq 0, \mathfrak{R}\left(D_{2}(y)\right)<0$. By continuity of the real part, which is a polynomial in $u$ and $v$, we have $\Re\left(D_{2}\left(y_{1}^{*}\right)\right) \leq 0$ and hence $y_{1} \leq y_{1}^{*} \leq y_{2}$. The right branch intersects the real axis at point $y_{2}^{*}$. For $y$ on this branch such that $\Im(y) \neq 0, \mathfrak{R}\left(D_{2}(y)\right)>0$ and by continuity of the real part, we have $\Re\left(D_{2}\left(y_{2}^{*}\right)\right) \geq 0$, which implies that $y_{2} \leq y_{2}^{*} \leq y_{3}$.

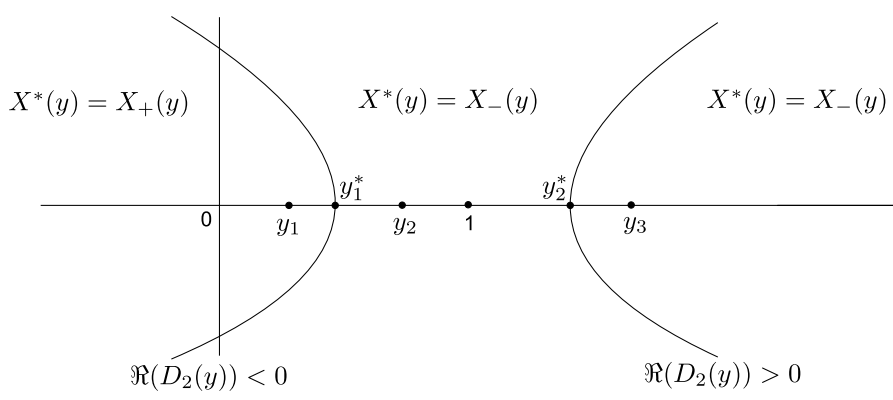

Fig. 1 Branches on which $\Im\left(D_{2}(y)\right)=0$ 
The function $X_{+}(y)$ is analytic in the domain $\left\{y: \Re(y) \leq y_{1}^{*}, \Im\left(D_{2}\left(y^{+}\right)\right)<\right.$ $0\} \cup\left(-\infty, y_{1}\right)$. The function $X_{-}(y)$ is analytic in the complementary domain of the closure of this set in $\mathbb{C} \backslash\left(\left[y_{1}, y_{2}\right] \cup\left[y_{3}, \infty\right)\right)$. To show that the function $X^{*}(y)$ is analytic in the whole of $\mathbb{C}$ deprived of the segments $\left[y_{1}, y_{2}\right]$ and $\left[y_{3}, \infty\right)$, by Moreira's theorem, it is sufficient to show that this function is continuous on the branch $\left\{y: \Im\left(D_{2}(y)\right)=0, \Re\left(D_{2}(y)\right) \leq 0\right\}$ separating the two above domains. But this is straightforwardly checked from the choice of the determination of the square root.

By using exactly the same arguments as in the proof of Lemma 1, we can prove the following result.

\section{Lemma 2 The function}

$$
X_{*}(y)= \begin{cases}X_{-}(y) \quad \text { when } y \in\left\{z: \Re(z) \leq y_{2}, \Im\left(D_{2}\left(z^{+}\right)\right)<0\right\} \cup\left(-\infty, y_{1}\right), \\ X_{+}(y) \text { otherwise }\end{cases}
$$

defined in $\mathbb{C} \backslash\left(\left[y_{1}, y_{2}\right] \cup\left[y_{3}, \infty\right)\right)$, is analytic.

We now turn to the functions $Y_{ \pm}(x)$. First note that $Y_{ \pm}(0)=0$. As shown in [32], when $x$ is close to the segment $\left[x_{1}, x_{2}\right], Y_{ \pm}(x)$ is close to a contour $\partial D_{y}$ in the $y$ plane included in the half-plane $\{y: \mathfrak{i}(y) \geq 0\}$. In particular, the point 0 lies in $\partial D_{y}$. In addition, when $y$ is close to the segment $\left[y_{1}, y_{2}\right], X(y)$ is in the $x$-plane close to a contour $\partial D_{x}$ surrounding the point 0 . The contours $\partial D_{x}$ and $\partial D_{y}$ delineate bounded open domains in the $x$-plane deprived of the segment $\left[x_{1}, x_{2}\right]$ and the $y$ plane deprived of the segment $\left[y_{1}, y_{2}\right]$ denoted by $D_{x}$ and $D_{y}$, respectively. Since our ultimate goal is to establish a conformal mapping between these two domains, and since $Y_{ \pm}(-i \varepsilon) \sim \pm(\cos (\pi / 4)+i \sin (\pi / 4)) \sqrt{\varepsilon /\left(r_{1} r_{2}\right)}$ for small $\varepsilon>0$, we are led to pick the function $Y_{+}(x)$ as a candidate for the desired conformal mapping because $Y_{+}(-i \varepsilon) \in D_{y}$ while $Y_{-}(-i \varepsilon) \notin D_{y}$ for sufficiently small $\varepsilon>0$.

\section{Lemma 3 The function}

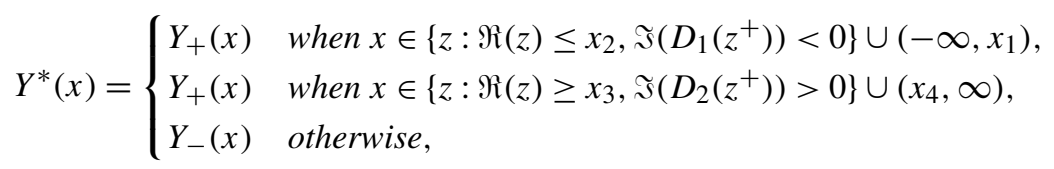

defined in $\mathbb{C} \backslash\left(\left[x_{1}, x_{2}\right] \cup\left[x_{3}, x_{4}\right]\right)$, is analytic.

Proof Let $x=u+i v$ with $u, v \in \mathbb{R}$. We have $D_{1}(x)=\Re\left(D_{1}(x)\right)+i \Im\left(D_{1}(x)\right)$ with

$$
\begin{aligned}
& \Re\left(D_{1}(x)\right)=\left((\hat{r}-u) u+v^{2}\right)^{2}-v^{2}(\hat{r}-2 u)^{2}-\frac{4 u}{r_{1} r_{2}}, \\
& \Im\left(D_{1}(x)\right)=2 v\left((\hat{r}-2 u) v^{2}+u(\hat{r}-u)(\hat{r}-2 u)-\frac{2}{r_{1} r_{2}}\right) .
\end{aligned}
$$


Note that $\mathfrak{s}\left(D_{1}(x)\right)=0$ if $(u, v)$ satisfies

$$
(2 u-\hat{r}) v^{2}=u(2 u-\hat{r})(u-\hat{r})-\frac{2}{r_{1} r_{2}} .
$$

Let $d_{1}(u)$ be the polynomial in the right-hand side of the above equation. This polynomial is of degree 3 and has at least one real root $\left(u_{1}\right.$ say). Since $\lim _{u \rightarrow+\infty} d_{1}(u)=$ $+\infty$ and $d_{1}(\hat{r})=-2 /\left(r_{1} r_{2}\right)<0, u_{1}>\hat{r}$. The polynomial $d_{1}(u)$ can then be decomposed as $d_{1}(u)=\left(u-u_{1}\right) d_{11}(u)$. If the polynomial $d_{11}(u)$ had no real roots, then this polynomial would be positive in the whole of $\mathbb{R}$ since $d_{1}(u)$ is positive for large $u$. When $u<\hat{r} / 2$, (9) would have two roots, namely

$$
v= \pm \sqrt{\frac{\left(u-u_{1}\right) d_{11}(u)}{2 u-\hat{r}}}
$$

We would then obtain two curves, one in the half-plane $\{x: \Im(x)>0\}$ and the other in the half-plane $\{x: \Im(x)<0\}$. Along each of these curves, the sign of $\Re\left(D_{1}(x)\right)$ should be constant (see the arguments in the proof of Lemma 1). But when $u \rightarrow-\infty$, $v^{2} \sim u^{2}$ and then $\Re\left(D_{1}(x)\right)<0$, and when $u \rightarrow \hat{r} / 2, v^{2} \sim-2 /\left(r_{1} r_{2}(2 u-\hat{r})\right)$ and $\Re\left(D_{1}(x)\right)>0$, which contradicts the fact that the sign of $\Re\left(D_{1}(x)\right)$ should be constant along the curves $\Im\left(D_{1}(x)\right)=0$. As a consequence, the polynomial $d_{1}(u)$ has three real roots. Let us denote these roots by $x_{1}^{*}, x_{2}^{*}$ and $x_{3}^{*}$ with $x_{1}^{*} \leq x_{2}^{*} \leq x_{3}^{*}$. Their product is equal to $1 /\left(r_{1} r_{2}\right)$ and since one of them is positive, the two others have the same sign.

We already know that $x_{3}^{*}>\hat{r}$. If $x_{1}^{*} \geq \hat{r} / 2$, then (9) defines two curves for $u \leq \hat{r} / 2$, one is included in the upper half-plane and the other in the lower half-plane, which is not possible for the same reasons as above. Hence, $x_{1}^{*} \leq \hat{r} / 2$. This also implies that $x_{2}^{*}<\hat{r} / 2$ since $d_{1}(\hat{r} / 2)=-2 /\left(r_{1} r_{2}\right)<0$. Hence, we have

$$
0 \leq x_{1}^{*} \leq x_{2}^{*}<\hat{r} / 2<\hat{r}<x_{3}^{*} .
$$

Let us consider the three curves defined by

$$
v= \pm \sqrt{\frac{\left(u-x_{1}^{*}\right)\left(u-x_{2}^{*}\right)\left(u-x_{3}^{*}\right)}{2 u-\hat{r}}} \quad \text { when } u \leq x_{1}^{*} \quad \text { or } x_{2}^{*} \leq u<\hat{r} / 2 \quad \text { or } u \geq x_{3}^{*} \text {. }
$$

See Fig. 2.

For the curve defined for $u \leq x_{1}^{*}$ it is easily checked that $\Re\left(D_{1}(x)\right)<0$ when $v \neq 0$ and by continuity we deduce that $\Re\left(D_{1}(x)\right) \leq 0$. This implies that $x_{1} \leq x_{1}^{*} \leq x_{2}$. Similar arguments show that $x_{3} \leq x_{3}^{*} \leq x_{4}$. For the curve defined for $x_{2} \leq u<\hat{r} / 2$, we have $\Re\left(D_{1}(x)\right)>0$ when $v \neq 0$ and hence $\Re\left(D_{1}(x)\right) \geq 0$ all along the curve. This implies that $x_{2} \leq x_{2}^{*} \leq x_{3}$. We finally have the ordering

$$
x_{1} \leq x_{1}^{*} \leq x_{2} \leq x_{2}^{*}<\hat{r} / 2<x_{3} \leq x_{3}^{*} \leq x_{4} .
$$

Note that it is easily checked that $x_{3}>\hat{r} / 2$. Indeed, if we assume that $x_{3} \leq \hat{r} / 2 \leq$ $x_{3}^{*} \leq x_{4}$, we would have $D_{1}(\hat{r} / 2) \leq 0$ and then $D_{1}(x)$ would be nonpositive for all 


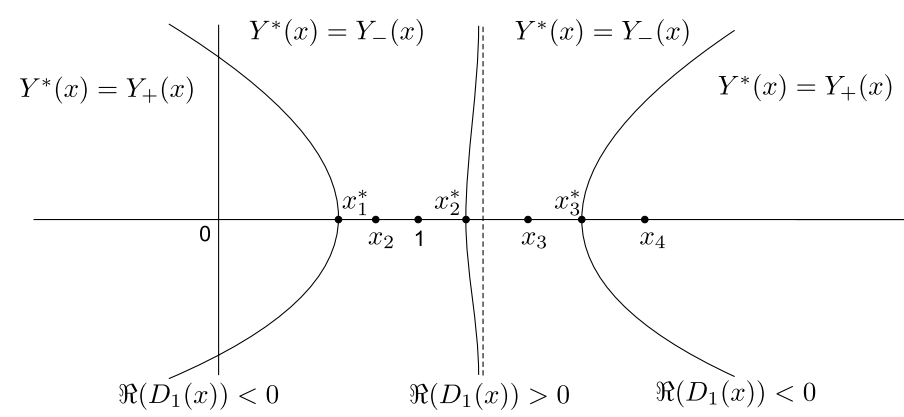

Fig. 2 Branches on which $\Im\left(D_{1}(x)\right)=0$

$x \geq \hat{r} / 2$ since the term $(\hat{r}-x) x$ is maximal at the point $\hat{r} / 2$; this is clearly not possible.

By invoking the same arguments as in the proof of Lemma 1, it is easily checked that the function $Y^{*}(x)$ defined by (8) is analytic in the complex plane deprived of the segments $\left[x_{1}, x_{2}\right]$ and $\left[x_{3}, x_{4}\right]$.

By similar arguments, we can prove the following result.

\section{Lemma 4 The function}

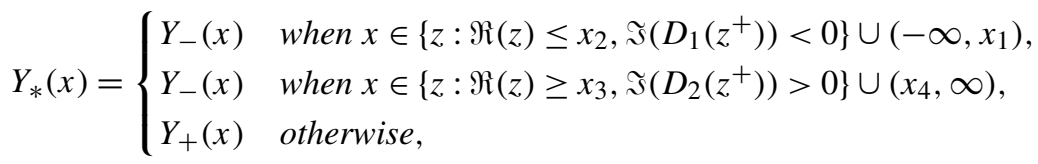

defined in $\mathbb{C} \backslash\left(\left[x_{1}, x_{2}\right] \cup\left[x_{3}, x_{4}\right]\right)$, is analytic.

To conclude this section, let us examine the images of the contours $\partial D_{x}$ and $\partial D_{y}$ by the analytic functions $Y^{*}$ and $X^{*}$, respectively. First note that for $x \in$ $\mathbb{C} \backslash\left(\left[x_{1}, x_{2}\right] \cap\left[x_{3}, x_{4}\right]\right), X^{*}\left(Y^{*}(x)\right)=x$ and for $y \in \mathbb{C} \backslash\left(\left[y_{1}, y_{2}\right] \cap\left[y_{3}, \infty\right)\right)$, $Y^{*}\left(X^{*}(y)\right)=y$. To prove the first equality, consider $x \in(-\infty, 0)$ sufficiently close to 0 , so that $Y^{*}(x)=Y_{+}(x) \sim \sqrt{-r_{1} x / r_{2}}$ and $X^{*}\left(Y^{*}(x)\right)=X_{+}\left(Y^{*}(x)\right) \sim x$. It follows that the equality $X^{*}\left(Y^{*}(x)\right)=x$ holds for a neighborhood of 0 and since the function $X^{*}\left(Y^{*}(x)\right)$ is analytic in $\mathbb{C} \backslash\left(\left[x_{1}, x_{2}\right] \cap\left[x_{3}, x_{4}\right]\right)$ this equality holds for the whole of $\mathbb{C} \backslash\left(\left[x_{1}, x_{2}\right] \cap\left[x_{3}, x_{4}\right]\right)$. Similar arguments can be invoked to prove the second equality.

Corollary 1 We have $X^{*}\left(\partial D_{y}\right) \subset\left[x_{1}, x_{2}\right]$ and $Y^{*}\left(\partial D_{x}\right) \subset\left[y_{1}, y_{2}\right]$.

Proof Consider $y \in \partial D_{y}$ (the case of $\partial D_{x}$ is similar). By construction, there exists an $x \in\left[x_{1}, x_{2}\right]$ such that

$$
y=Y_{+}(x+0 i), \quad \bar{y}=Y_{+}(x-0 i), \quad y=Y_{-}(x-0 i), \quad \bar{y}=Y_{-}(x+0 i) .
$$

Note that we use the notation $x+0 i$ (resp. $x-0 i$ ) to designate the limit of a sequence in the upper (resp. lower) half-plane converging to $x \in \mathbb{R}$. From the definition of 
$Y^{*}(x)$, the determination of this function at the point $x \pm 0 i$ is either $Y_{+}(x \pm 0 i)$ or $Y_{-}(x \pm 0 i)$. It follows that $y=Y^{*}(x+\varepsilon 0 i)$ where $\varepsilon= \pm 1$, depending on the determination of $Y^{*}(x)$. It follows that $X^{*}(y)=X^{*}\left(Y^{*}(x+\varepsilon 0 i)\right)=x \in\left[x_{1}, x_{2}\right]$. Hence, $X^{*}\left(\partial D_{y}\right) \subset\left[x_{1}, x_{2}\right]$.

\subsection{Conformal mappings}

We are now able to establish the conformal mappings which will play a crucial role in the derivation of the boundary functions $P(0, y)$ and $P(x, 0)$.

Proposition 1 The function $X^{*}(y)$ is a conformal mapping from $D_{y}$ onto $D_{x}$. The reciprocal function is $Y^{*}(x)$.

Proof As noted before, when $y$ is in $D_{y}$ and sufficiently close to $0, X_{+}(y) \equiv$ $X^{*}(y) \in D_{x}$. Since the set $D_{y}$ is an open and simply connected domain and since $X^{*}(y)$ is an analytic function, $X^{*}\left(D_{y}\right) \cap D_{x}$ is a non-null, open and simply connected domain included in $D_{x}$.

If $D_{x}$ is not a subset of $X^{*}\left(D_{y}\right)$, consider the complementary set $X^{*}\left(D_{y}\right)^{c} \cap D_{x} \neq$ $\emptyset$ in $D_{x}$. Let $x$ be a point on the boundary between this set and $X^{*}\left(D_{y}\right) \cap D_{x}$. There exist a sequence $\left(x_{n}\right)$ in $X^{*}\left(D_{y}\right) \cap D_{x}$, and a sequence $\left(x_{n}^{\prime}\right)$ in the interior of $X^{*}\left(D_{y}\right)^{c} \cap D_{x}$ both converging to $x$. Since $\left(x_{n}\right)$ is in $X^{*}\left(D_{y}\right) \cap D_{x}$, there exists a sequence $\left(y_{n}\right)$ in $D_{y}$ such that $X^{*}\left(y_{n}\right)=x_{n}$. Moreover, as we have $X^{*}\left(Y^{*}(x)\right)=x$ for all $x$ in the $x$-plane deprived of the segments $\left[x_{1}, x_{2}\right]$ and $\left[x_{3}, x_{4}\right]$, and $Y^{*}\left(X^{*}(y)\right)=y$ for all $y$ in the $y$-plane deprived of the segments $\left[y_{1}, y_{2}\right]$ and $\left[y_{3}, \infty\right)$, the sequences $\left(y_{n}\right)$ and $\left(Y^{*}\left(x_{n}\right)\right)$ converge to the same point. But by definition the points $Y^{*}\left(x_{n}\right)$ lie outside the domain $D_{y}$. It follows that these two sequences converge to a point on $\partial D_{y}$. By Corollary 1 , this implies that $x \in\left[x_{1}, x_{2}\right]$, which is not possible. It follows that $D_{x} \subset X^{*}\left(D_{y}\right)$.

If the above inclusion is strict, we consider a point $x$ on the boundary $\partial D_{x}$. There should exist a point $y$ in $D_{y}$ such that $X^{*}(y)=x$ but this is not possible since $y$ should be in $\left[y_{1}, y_{2}\right]$ since $Y^{*}\left(\partial D_{x}\right) \subset\left[y_{1}, y_{2}\right]$. It follows that $X^{*}\left(D_{y}\right)=D_{x}$. In addition, the function $X^{*}(y)$ is one to one since $Y^{*}\left(X^{*}(y)\right)=y$. It follows that this function is a conformal mapping from $D_{y}$ onto $D_{x}$ and the reciprocal function is $Y^{*}$.

The conformal mappings $X^{*}$ and $Y^{*}$ between the domains $D_{x} \backslash\left[x_{1}, x_{2}\right]$ and $D_{y} \backslash$ $\left[y_{1}, y_{2}\right]$ are illustrated in Fig. 3. While $X^{*}$ maps $D_{y} \backslash\left[y_{1}, y_{2}\right]$ onto $D_{x} \backslash\left[x_{1}, x_{2}\right]$, the set $X_{*}\left(D_{y} \backslash\left[y_{1}, y_{2}\right]\right)$ is an open domain surrounding $D_{x}$ in the $x$-plane. Similarly, $Y_{*}\left(D_{x} \backslash\left[x_{1}, x_{2}\right]\right)$ is an open domain surrounding $D_{y}$ in the $y$-plane.

It is worth noting that $X^{*}(\xi) \rightarrow x \in \partial D_{x}$ from inside $D_{x}$ when $\xi \rightarrow y \in\left[y_{1}, y_{2}\right]$. Similarly, $Y^{*}(\xi) \rightarrow y \in \partial D_{y}$ from inside $D_{y}$ when $\xi \rightarrow x \in\left[x_{1}, x_{2}\right]$. We also have $X_{*}(\xi) \rightarrow x \in \partial D_{x}$ from outside $D_{x}$ when $\xi \rightarrow y \in\left[y_{1}, y_{2}\right]$ and $Y_{*}(\xi) \rightarrow y \in \partial D_{y}$ from outside $D_{y}$ when $\xi \rightarrow x \in\left[x_{1}, x_{2}\right]$.

\section{Intersection points of the curves $h_{1}(x, y)=0$ and $h_{2}(x, y)=0$}

When $h_{1}(x, y)=0$, we see from (1) that we can express $P(x, 0)$ (resp. $P(0, y)$ ) as a function of $P(0, y)$ (resp. $P(x, 0))$ and $h_{4}(x, y)$, where the function $h_{2}(x, y)$ 
$y$-plane

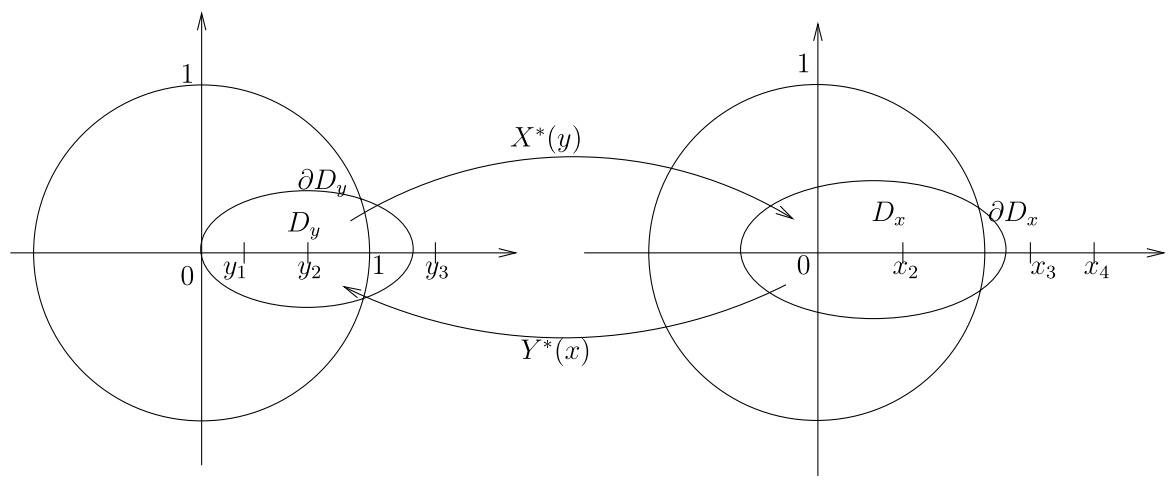

Fig. 3 Fundamental domains $D_{y}$ and $D_{x}$

appears in the denominator. The common solutions of the equations $h_{1}(x, y)=0$ and $h_{2}(x, y)=0$ are then potential singularities for the functions $P(x, 0)$ and $P(0, y)$.

\subsection{The common roots in variable $y$}

Let $y \in \mathbb{C} \backslash\left(\left[y_{1}, y_{2}\right] \cup\left[y_{3}, \infty\right)\right)$ and $h_{1}(x, y)=0, x=X_{ \pm}(y)$. If in addition $h_{2}(x, y)=0$, then $y$ is a root of the resultant in $x$ of the two polynomials $h_{1}(x, y)$ and $h_{2}(x, y)$ (see the Appendix); this resultant, denoted by $Q_{x}(y)$, is a polynomial of degree 5 in $y$, which has at most four distinct zeros in $\mathbb{C}$. The point 0 is a double root. Another trivial root is 1 since $h_{1}(1,1)=0$ and $h_{2}(1,1)=0$. As shown in the Appendix, the resultant $Q_{x}(y)$ can actually be decomposed as

$$
Q_{x}(y)=c_{x} y^{2}(y-1) \mathcal{Q}_{x}(y),
$$

where $\mathcal{Q}_{x}(y)$ is the quadratic polynomial

$$
\mathcal{Q}_{x}(y)=\lambda v_{1} y^{2}+v_{2}\left(v_{2}-v_{1}+\lambda\right) y-v_{2}^{2}
$$

and $c_{x}$ is a constant.

When $y$ describes the segment $\left[y_{2}, y_{3}\right]$, the curves $y \rightarrow x=X_{ \pm}(y)$ describe the contour of a closed domain $\Omega_{y}$ in the $(y, x)$-plane as illustrated in Fig. 4 . The contour $\partial \Omega_{y}$ of $\Omega_{y}$ contains the point $(1,1)$.

When $h_{2}(x, y)=0$,

$$
x=\frac{v_{1} y^{2}}{\left(v_{1}-v_{2}\right) y+v_{2}} .
$$

As illustrated in Fig. 4, when $r_{1}<1$, the hyperbolic branch defined by (12) intersects the branch $x=X_{-}(y)$ at some point with a negative abscissa. The same observation is true when $r_{1} \geq 1$. It follows that the resultant $Q_{y}(x)$ has four real roots and the 
Fig. 4 Intersection points of the functions $X_{ \pm}(y)$ and the curve $h_{2}(x, y)=0$ when $r_{1}<1$

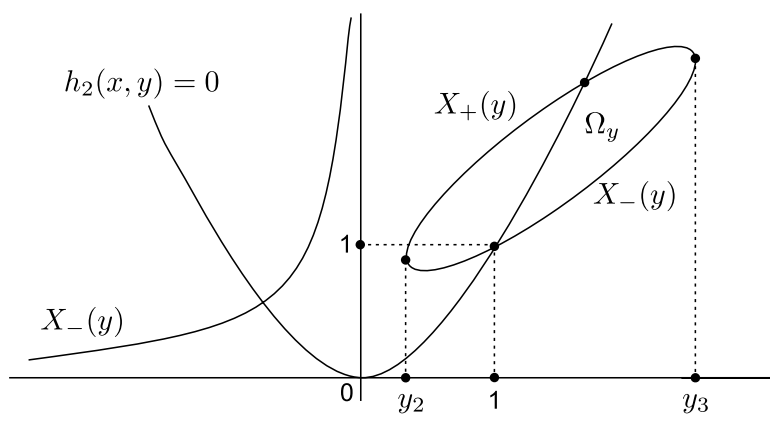

quadratic polynomial $\mathcal{Q}_{x}(y)$ has two real roots, one is negative and the other is in $\left[y_{2}, y_{3}\right]$. The positive root is (with $\rho_{i}=\lambda / v_{i}$ )

$$
y^{*}=\frac{\rho_{1}}{2 \rho_{2}}\left(\frac{1}{\rho_{1}}-\frac{1}{\rho_{2}}-1+\sqrt{\left(\frac{1}{\rho_{1}}-\frac{1}{\rho_{2}}-1\right)^{2}+\frac{4}{\rho_{1}}}\right)
$$

and the negative root is

$$
y_{*}=\frac{\rho_{1}}{2 \rho_{2}}\left(\frac{1}{\rho_{1}}-\frac{1}{\rho_{2}}-1-\sqrt{\left(\frac{1}{\rho_{1}}-\frac{1}{\rho_{2}}-1\right)^{2}+\frac{4}{\rho_{1}}}\right) \text {. }
$$

It is worth noting that $y^{*}$ does not depend on $p$. From the Appendix, we know that $y^{*} \in\left(1, y_{3}\right]$.

\subsection{The common roots in variable $x$}

The resultant in $x$ of the polynomials $h_{1}(x, y)$ and $h_{2}(x, y)$ is a polynomial of degree 5 with trivial roots 0 and 1 ( 0 is a double root). If $x \neq 0$ and $(x, y)$ is an intersection point of the curves $h_{1}(x, y)=0$ and $h_{2}(x, y)=0$, then

$$
y=\frac{v_{2}}{\lambda+v_{2}-\lambda x} .
$$

For $x \in\left[x_{2}, x_{3}\right]$, the curves $y=Y_{ \pm}(x)$ delineate a closed domain $\Omega_{x}$ such that its contour $\partial \Omega_{x}$ contains the point $(1,1)$. Note that if $r_{1}<r_{2}$, then $Y_{+}(1)=1$ and if $r_{1}>r_{2}$, then $Y_{-}(1)=1$.

The hyperbolic branch defined by (14) intersects the branch $y=Y_{-}(x)$ or $y=$ $Y_{+}(x)$ at a point with abscissa $x>x_{4}$. It follows that the resultant in $y$ of the polynomials $h_{1}(x, y)$ and $h_{2}(x, y)$, denoted by $Q_{y}(x)$, can be decomposed as

$$
Q_{y}(x)=c_{y} x^{2}(x-1) \mathcal{Q}_{y}(x),
$$

where $c_{y}$ is a constant and

$$
\mathcal{Q}_{y}(x)=\lambda^{2} x^{2}-\left(\lambda+v_{1}+v_{2}\right) \lambda x+v_{1} v_{2}
$$


The roots $x^{*}$ and $x_{*}$ are given by

$$
x^{*}=\frac{1}{2}\left(1+\frac{1}{\rho_{1}}+\frac{1}{\rho_{2}}-\sqrt{\left(1+\frac{1}{\rho_{1}}+\frac{1}{\rho_{2}}\right)^{2}-\frac{4}{\rho_{1} \rho_{2}}}\right)
$$

and

$$
x_{*}=\frac{1}{2}\left(1+\frac{1}{\rho_{1}}+\frac{1}{\rho_{2}}+\sqrt{\left(1+\frac{1}{\rho_{1}}+\frac{1}{\rho_{2}}\right)^{2}-\frac{4}{\rho_{1} \rho_{2}}}\right),
$$

and are such that $x^{*} \leq x_{3}<x_{4} \leq x_{*}$. In addition, we know that $x^{*}>1$ and hence $x^{*} \in\left(1, x_{3}\right]$. The variable $x^{*}$ does not depend on the probability $p$.

From the above observations, we deduce the following result.

Proposition 2 The equation $Q_{y}\left(X^{*}(y)\right)=0$ has a solution in $\left(-\infty, y_{3}\right]$, which is necessarily equal to $y^{*} \in\left(1, y_{3}\right]$, if and only if $x^{*}=X_{-}\left(y^{*}\right)$.

Similarly, the equation $Q_{x}\left(Y^{*}(x)\right)=0$ has a solution in $\left(-\infty, x_{3}\right]$, which is necessarily equal to $x^{*} \in\left(1, x_{3}\right]$, if and only if $y^{*}=Y_{-}\left(x^{*}\right)$.

It is worth noting that we can have $x^{*}=X^{*}\left(y^{*}\right)$ only if $1=X^{*}(1)$, that is $r_{1} \leq 1$. Similarly, we can have $y^{*}=Y^{*}\left(x^{*}\right)$ only if $1=Y^{*}(1)$, that is $r_{1} \geq r_{2}$.

\section{Boundary value problems}

We first determine the function $P(x, 0)$; the derivation of the function $P(0, y)$ can be done in the exact same fashion.

Proposition 3 The function $P(x, 0)$ is given by

$$
P(x, 0)= \begin{cases}\frac{1}{2 \pi i} \int_{\partial D_{x}} \frac{g_{x}(z)}{z-x} d z & \text { for } x \in D_{x}, \\ g_{x}(x)+\frac{1}{2 \pi i} \int_{C_{x}} \frac{g_{x}(z)}{z-x} d z & \text { for } x \in \mathbb{C} \backslash D_{x},\end{cases}
$$

where $C_{x}$ is a contour in $D_{x}$ surrounding the slit $\left[x_{1}, x_{2}\right]$ and such that the function $g_{x}$ given by

$$
g_{x}(x)=(1-\rho) \frac{\nu_{2} Y^{*}(x)\left(p v_{1} Y^{*}(x)-\lambda x^{2}\right)}{(1-p) x \mathcal{Q}_{x}\left(Y^{*}(x)\right)}
$$

is analytic in the strip delineated by the contours $C_{x}$ and $\partial D_{x}$. The function $P(x, 0)$ is a meromorphic function in $\mathbb{C} \backslash\left[x_{3}, x_{4}\right]$ with singularities at the solutions to the equation $\mathcal{Q}_{x}\left(Y^{*}(x)\right)=0$, if they exist.

Proof From the analysis carried out in Sect. 2, we know that for $y$ in a neighborhood $V_{y}(0)$ of $0^{+}, X^{*}(y)$ is close to 0 in $D_{x}(0,1)$ (the unit disk in the $x$-plane). For $y \in$ 
$V_{y}(0)$, we deduce from (1) that

$$
h_{2}\left(X^{*}(y), y\right) P\left(X^{*}(y), 0\right)+h_{3}\left(X^{*}(y), y\right) P(0, y)+h_{4}\left(X^{*}(y), y\right) P(0,0)=0,
$$

which implies that

$$
P\left(X^{*}(y), 0\right)=\frac{p}{1-p} P(0, y)-(1-\rho) \frac{h_{4}\left(X^{*}(y), y\right)}{h_{2}\left(X^{*}(y), y\right)} .
$$

Note that $h_{2}\left(X^{*}(y), y\right)=0$ if and only if $Q_{y}\left(X^{*}(y)\right)=0$, which has only real solutions (see Sect. 3). From Proposition 2, this equation has a solution in $\left(-\infty, y_{3}\right]$ if and only if $x^{*}=X^{*}\left(y^{*}\right)$, which is then the unique solution and which is in $\left(1, y_{3}\right]$. If $\alpha=Y^{*}\left(x_{2}\right) \leq 1$, the domain $D_{y}$ is included in the unit disk $D_{y}(0,1)$ and in that case the function $h_{4}\left(X^{*}(y), y\right) / h_{2}\left(X^{*}(y), y\right)$ has no singularities in $D_{y}$. If $\alpha>1$, then $r_{1}>r_{2}$. In this case, $x^{*}$ is not equal to $X^{*}\left(y^{*}\right)$ and the function $h_{4}\left(X^{*}(y), y\right) / h_{2}\left(X^{*}(y), y\right)$ has no singularities in $D_{y}$. Hence, by using the same arguments as in [32], we deduce that the function $P(x, 0)$ can be analytically continued to the domain $D_{x}$. (We use the fact that the function $P(x, 0)$ can be expanded in a power series of $x$ at the point 0 with positive coefficients and $P\left(0, y_{2}\right)<\infty$, which implies that $P(x, 0)$ is analytic in the disk with center 0 and radius $X^{*}\left(y_{2}\right)$ containing $D_{x}$.)

Now, if we use the function $X_{*}(y)$, we obtain a meromorphic function in a domain surrounding from outside the domain $D_{x}$. If we take $y$ in a sufficiently small neighborhood of $\left[y_{1}, y_{2}\right]$ we can analytically define $P(x, 0)$ in an outer neighborhood of $D_{x}$.

Consider $x_{0} \in \partial D_{x}$. Then there exists $y_{0} \in\left[y_{1}, y_{2}\right]$ such that $X^{*}(y) \rightarrow x_{0}$ from inside when $y \rightarrow y_{0}$. In that case, $X_{*}(y) \rightarrow \bar{x}_{0}$ from outside. Let us define the interior (resp. exterior) limit $P_{i}(x, 0)$ (resp. $\left.P_{e}(x, 0)\right)$ of the function $P(x, 0)$ with respect to the contour $\partial D_{y}$ by

$$
P_{i}\left(x_{0}, 0\right)=\lim _{x \rightarrow x_{0}, x \in D_{x}} P(x, 0)\left(\text { resp. } P_{e}\left(x_{0}, 0\right)=\lim _{x \rightarrow x_{0}, x \in \mathbb{C} \backslash D_{x}} P(x, 0)\right) .
$$

We then deduce from the above observation that for $x \in \partial D_{y}$ and $y=Y^{*}(x)$

$$
P_{i}(x, 0)=\frac{p P(0, y)}{1-p}-(1-\rho) \frac{h_{4}(x, y)}{h_{2}(x, y)}, \quad P_{e}(x, 0)=\frac{p P(0, y)}{1-p}-(1-\rho) \frac{h_{4}(\bar{x}, y)}{h_{2}(\bar{x}, y)},
$$

since $P(\cdot, 0), h_{2}$ and $h_{4}$ have real coefficients. Hence, we arrive at the fact that for $x \in \partial D_{x}$ and $y=Y^{*}(x)$

$$
P_{i}(x, 0)-P_{e}(x, 0)=-2 i(1-\rho) \Im\left(\frac{h_{4}(x, y)}{h_{2}(x, y)}\right) .
$$

Note that for $x \in \partial D_{x}$, we have $x \bar{x}=y / r_{1}=Y^{*}(x) / r_{1}$ since $x$ and $\bar{x}$ are the two solutions to (1) in $x$. In addition, from the Appendix, we know that the resultant $Q_{x}(y)$ can be written as

$$
Q_{x}(y)=p_{x}(x, y) h_{1}(x, y)+q_{x}(x, y) h_{2}(x, y),
$$


where $p_{x}(x, y)$ and $q_{x}(x, y)$ are polynomials in $x$ and $y$. For $y=Y^{*}(x)$, we have $h_{1}(x, y)=0$ and then

$$
Q_{x}(y)=q_{x}(x, y) h_{2}(x, y) .
$$

Simple computations show that

$$
\frac{h_{4}(x, y)}{h_{2}(x, y)}=-1+\frac{\nu_{2} x(y-1)}{h_{2}(x, y)}
$$

and

$$
q_{x}(x, y)=\lambda y b_{1}(y) x-\left(\lambda(1-p) v_{1} y^{3}+a_{1}(y) b_{1}(y)\right),
$$

where

$$
\begin{aligned}
& a_{1}(y)=\left(\lambda+p v_{1}+(1-p) v_{2}\right) y-(1-p) \nu_{2}, \\
& b_{1}(y)=(1-p)\left(\left(v_{2}-v_{1}\right) y-v_{2}\right) .
\end{aligned}
$$

Hence, for $x \in \partial D_{y}$ and $y=Y^{*}(x)$, we have

$$
\Im\left(\frac{h_{4}(x, y)}{h_{2}(x, y)}\right)=\Im\left(\frac{\nu_{2} x(y-1)\left(\lambda y b_{1}(y) x-\left(\lambda(1-p) \nu_{1} y^{3}+a_{1}(y) b_{1}(y)\right)\right)}{-v_{1}(1-p)^{2} y^{2}(y-1) \mathcal{Q}_{x}(y)}\right) .
$$

By using the fact that $\lambda y x^{2}-a_{1}(y) x=-p v_{1} y^{2}$, we have

$$
\Im\left(\frac{h_{4}(x, y)}{h_{2}(x, y)}\right)=\Im\left(\frac{\nu_{2}\left(p v_{1} b_{1}(y)+\lambda(1-p) \nu_{1} y x\right)}{\nu_{1}(1-p)^{2} \mathcal{Q}_{x}(y)}\right)
$$

and then

$$
\Im\left(\frac{h_{4}(x, y)}{h_{2}(x, y)}\right)=\frac{v_{2} \lambda y \Im(x)}{(1-p) \mathcal{Q}_{x}(y)}=\frac{\nu_{2} \lambda y\left(r_{1} x^{2}-y\right)}{2 i r_{1} x(1-p) \mathcal{Q}_{x}(y)} .
$$

We finally arrive at the classical Riemann-Hilbert problem. For $x \in \partial D_{x}$,

$$
P_{i}(x, 0)-P_{e}(x, 0)=(1-\rho) \frac{\nu_{2} Y^{*}(x)\left(p \nu_{1} Y^{*}(x)-\lambda x^{2}\right)}{x(1-p) \mathcal{Q}_{x}\left(Y^{*}(x)\right)}=g_{x}(x) .
$$

The solution to this Riemann-Hilbert problem is given by

$$
P(x, 0)=\frac{1}{2 \pi i} \int_{\partial D_{x}} \frac{g_{x}(z)}{z-x} d z \quad \text { for } x \notin \partial D_{x} .
$$

The above formula defines an analytic function in $D_{x}$. For $x \in \mathbb{C} \backslash D_{x}$, let us pick a closed contour $C_{x}$ in $D_{x}$ surrounding the slit $\left[x_{1}, x_{2}\right]$ and so that the function $g_{x}$ is analytic in the strip delineated by the contours $\partial D_{x}$ and $C_{x}$. Then, we have

$$
\frac{1}{2 \pi i} \int_{\partial D_{x}} \frac{g_{x}(z)}{z-x} d z=g_{x}(x)+\frac{1}{2 \pi i} \int_{C_{x}} \frac{g_{x}(z)}{z-x} d z .
$$


The function on the right-hand side of the above equation defines a meromorphic function in $\mathbb{C} \backslash\left[x_{3}, x_{4}\right]$.

We can replace the integrals appearing in (17) with integrals along the segment $\left[y_{1}, y_{2}\right]$. We then obtain elliptic integrals. Since these integrals do not appear as simple combinations of Jacobi elliptic functions, we do not further investigate the connection between the function $P(x, 0)$ and elliptic functions. Finally, it is worth noting that the radius of convergence of the function $P(x, 0)$ is equal to either $x_{3}$ or else $x^{*}$ if $y^{*}=Y^{*}\left(x^{*}\right)$.

By adapting the above proof to the function $P(0, y)$, we obtain the following result.

Proposition 4 The function $P(0, y)$ is given by

$$
P(0, y)= \begin{cases}\frac{1}{2 \pi i} \int_{\partial D_{y}} \frac{g_{y}(z)}{z-y} d z & \text { for } y \in D_{y}, \\ g_{y}(y)+\frac{1}{2 \pi i} \int_{C_{y}} \frac{g_{y}(z)}{z-y} d z & \text { for } y \in \mathbb{C} \backslash D_{y},\end{cases}
$$

where $C_{y}$ is a closed contour in $D_{y}$ surrounding the slit $\left[y_{1}, y_{2}\right]$ such that the function $g_{y}$ given by

$$
g_{y}(y)=(1-\rho) \frac{\lambda\left(p v_{1} y^{2}-(1-p) \nu_{2} X^{*}(y)\right)}{p y \mathcal{Q}_{y}\left(X^{*}(y)\right)}
$$

is analytic in the strip delineated by the contours $C_{y}$ and $\partial D_{y}$. The function $P(0, y)$ is a meromorphic function in $\mathbb{C} \backslash\left[y_{3}, y_{4}\right]$ with singularities at the solutions to the equation $\mathcal{Q}_{y}\left(X^{*}(y)\right)=0$, if they exist.

Proof Denote by $P_{i}(0, y)$ and $P_{e}(0, y)$ the interior and exterior limits of the function $P(0, y)$ with respect to the contour $\partial D_{y}$. We have for $y \in \partial D_{y}$ and $x=X^{*}(y)$

$$
P_{i}(0, y)-P_{e}(0, y)=2 i(1-\rho) \frac{1-p}{p} \mathfrak{s}\left(\frac{h_{4}(x, y)}{h_{2}(x, y)}\right) .
$$

We have $Q_{y}(x)=q_{y}(x, y) h_{2}(x, y)$ for $x=x^{*}(y)$ with

$$
\begin{aligned}
q_{y}(x, y)= & (1-p) v_{1}\left[-y p v_{1}\left(p\left(v_{2}-v_{1}\right) x+\alpha_{1}(x)\right)\right. \\
& \left.+p \alpha_{1}(x)\left(v_{2}-v_{1}\right) x+\alpha_{1}(x)^{2}-p v_{1} v_{2} x\right] .
\end{aligned}
$$

Then

$$
\Im\left(\frac{h_{4}(x, y)}{h_{2}(x, y)}\right)=\frac{v_{2} x}{Q_{y}(x)} \Im\left((y-1) q_{y}(x, y)\right)=\frac{\lambda\left(p v_{1} y^{2}-(1-p) v_{2} x\right)}{2 i(1-p) y \mathcal{Q}_{y}(x)},
$$

which implies that

$$
P_{i}(0, y)-P_{e}(0, y)=(1-\rho) \frac{\lambda\left(p v_{1} y^{2}-(1-p) v_{2} x\right)}{p y \mathcal{Q}_{y}(x)}
$$




$$
=(1-\rho) \frac{\lambda\left(p v_{1} y^{2}-(1-p) \nu_{2} X^{*}(y)\right)}{p y \mathcal{Q}_{y}\left(X^{*}(y)\right)}=g_{y}(y) \text {. }
$$

Note that 0 is a removable singularity of the function $g_{y}(y)$ since $X^{*}(y) \sim-r_{2} y^{2} / r_{1}$ when $y \rightarrow 0$.

\section{Asymptotic analysis}

We derive in this section the tail of the distribution of the numbers of customers in the first and the second queue. For this purpose, we consider the generating functions $P(x, 1)$ and $P(1, y)$, which satisfy

$$
P(x, 1)=\sum_{n=0}^{\infty} \mathbb{P}\left(N_{1}=n\right) x^{n} \quad \text { and } \quad P(1, y)=\sum_{n=0}^{\infty} \mathbb{P}\left(N_{2}=n\right) y^{n},
$$

where $N_{1}$ and $N_{2}$ are the numbers of customers in the first and the second queue, respectively. From (1), we have

$$
P(x, 1)=v_{1} \frac{(1-p) P(x, 0)-p P(0,1)-(1-p)(1-\rho)}{\lambda x-p v_{1}}
$$

and

$$
P(1, y)=\frac{\left(v_{1} y+v_{2}\right)((1-p) P(1,0)-p P(0, y)-(1-p)(1-\rho))+v_{2}(1-\rho)}{(1-p) v_{2}-p v_{1} y} .
$$

Note that the normalizing condition $P(1,1)=1$ implies that

$$
(1-p) P(1,0)-p P(0,1)=(1-p)(1-\rho)+\rho_{1}-p .
$$

Lemma 5 If $r_{2} \leq 1$, then

$$
(1-p) P\left(r_{1}^{-1}, 0\right)-p P(0,1)-(1-p)(1-\rho)=0,
$$

which implies that the point $1 / r_{1}$ is a removable singularity for the function $P(x, 1)$. If $r_{2}>1$ (and then $r_{1} \leq 1$ by the stability condition (2)), we have

$$
(1-p) P\left(r_{1}^{-1}, 0\right)-p P(0,1)-(1-p)(1-\rho)<0
$$

and the point $1 / r_{1}$ is a nonremovable singularity for the function $P(x, 1)$.

Proof We know that $P(x, 0)$ is a meromorphic function in the disk with center 0 and radius $x_{3}$, with a unique potential singularity at point $x^{*}$. Equation (1) implies for $x \neq x^{*}$, when $x^{*}$ is a singularity for $P(x, 0)$,

$$
h_{2}\left(x, Y^{*}(x)\right) P(x, 0)+h_{3}\left(x, Y^{*}(x)\right) P\left(0, Y^{*}(x)\right)+h_{4}\left(x, Y^{*}(x)\right)=0 .
$$


When $r_{2} \leq 1$, we have $Y^{*}\left(1 / r_{1}\right)=1$ and the above equation implies (19). When $r_{2}>1$ (and hence $\left.r_{1} \leq 1\right)$, we have $Y^{*}\left(1 / r_{1}\right)=1 / r_{2}<1$, and (21) implies

$$
\begin{aligned}
& (1-p) P\left(1 / r_{1}, 0\right)-p P\left(0,1 / r_{2}\right)-(1-p)(1-\rho) \\
& =(1-\rho) \frac{\frac{\nu_{2}}{r_{1}}\left(1-\frac{1}{r_{2}}\right)}{\frac{\nu_{1}}{r_{2}}\left(\frac{1}{r_{2}}-\frac{1}{r_{1}}\right)+\frac{\nu_{2}}{r_{1}}\left(\frac{1}{r_{2}}-1\right)}<0 .
\end{aligned}
$$

Since $P\left(0,1 / r_{2}\right) \leq P(0,1)$, Inequality (20) follows.

Similar arguments yield the following result for the function $P(1, y)$; the proof is omitted.

Lemma 6 We have

$$
(1-p) P(1,0)-p P\left(0, r_{1} / r_{2}\right)-(1-p)(1-\rho)+p(1-\rho)=0,
$$

and hence the point $r_{1} / r_{2}$ is a removable singularity for the function $P(1, y)$.

By using the two lemmas above, we are now able to determine the tails of the probability distributions of the random variables $N_{1}$ and $N_{2}$.

Proposition 5 For given system parameters $\lambda, v_{1}, v_{2}$ and $p$, the exact asymptotics of $\mathbb{P}\left(N_{1}=n\right)$ as $n \rightarrow \infty$ are:

I If $y^{*}=Y^{*}\left(x^{*}\right)$ and $x^{*}<x_{3}$, which can occur only if $r_{1} \geq r_{2}$, then

$$
\mathbb{P}\left(N_{1}=n\right) \sim \kappa_{1}^{(1)}\left(\frac{1}{x^{*}}\right)^{n} .
$$

II If $y^{*} \neq Y^{*}\left(x^{*}\right)$ and $r_{2}>1$ (and then $\left.r_{1} \leq 1\right)$,

$$
\mathbb{P}\left(N_{1}=n\right) \sim \kappa_{2}^{(1)}\left(r_{1}\right)^{n} .
$$

III If $y^{*} \neq Y^{*}\left(x^{*}\right)$ and $r_{2} \leq 1,1 / r_{1}$ is a removable singularity for $P(x, 1)$ and we have

$$
\mathbb{P}\left(N_{1}=n\right) \sim \kappa_{3}^{(1)} \frac{1}{n \sqrt{n}}\left(\frac{1}{x_{3}}\right)^{n} .
$$

IV If $y^{*}=Y^{*}\left(x_{3}\right)$ and $x^{*}=x_{3}$,

$$
\mathbb{P}\left(N_{1}=n\right) \sim \kappa_{4}^{(1)} \frac{1}{\sqrt{n}}\left(\frac{1}{x^{*}}\right)^{n},
$$

with $x_{3}$ the third largest real root of $D_{1}(x)$ in (4), and $Y^{*}(x), y^{*}, x^{*}$ as in (8), (13), (16), respectively. Further, $r_{i}=\lambda /\left(p v_{i}\right), \rho=1-\lambda / \nu_{1}-\lambda / \nu_{2}$ and

$$
\kappa_{1}^{(1)}=\frac{\nu_{1} v_{2}(1-\rho)\left((1-p) \nu_{2} x^{*}-p v_{1}\left(y^{*}\right)^{2}\right)}{\left(\lambda x^{*}-p v_{1}\right)\left(v_{2}^{2}+\lambda v_{1}\left(y^{*}\right)^{2}\right) x^{*}},
$$




$$
\begin{aligned}
\kappa_{2}^{(1)} & =P(0,1)+\frac{1-p}{p}\left(1-\rho-P\left(r_{1}^{-1}, 0\right)\right), \\
\kappa_{3}^{(1)} & =\frac{(1-\rho) \lambda v_{1} v_{2}}{4 \sqrt{\pi}\left(\lambda x_{3}-p v_{1}\right)} \frac{\frac{\lambda^{2}(1-p)}{p v_{2}} x_{3}^{2}+2 \lambda x_{3}-\left(p \lambda+v_{1}\right)}{\mathcal{Q}_{y}\left(x_{3}\right) \mathcal{Q}_{y}^{*}\left(x_{3}\right)} \sqrt{x_{3}} \tau_{x}, \\
\kappa_{4}^{(1)} & =\frac{(1-\rho) \lambda v_{1} v_{2}}{2 \sqrt{\pi}\left(\lambda x_{3}-p v_{1}\right)} \frac{\frac{\lambda^{2}(1-p)}{p v_{2}} x_{3}^{2}+2 \lambda x_{3}-\left(p \lambda+v_{1}\right)}{\sqrt{x_{3}} \mathcal{Q}_{y}^{\prime}\left(x_{3}\right) \mathcal{Q}_{y}^{*}\left(x_{3}\right)} \tau_{x},
\end{aligned}
$$

with $\mathcal{Q}_{y}(x)$ as in (15),

$$
\tau_{x}=\sqrt{\left(x_{3}-x_{1}\right)\left(x_{3}-x_{2}\right)\left(x_{4}-x_{3}\right)}
$$

and

$$
\mathcal{Q}_{y}^{*}(x)=\frac{1}{\lambda^{2}}\left(x-\frac{p v_{1} y^{*}}{x^{*}}\right)\left(x-\frac{p v_{1} y_{*}}{x_{*}}\right) .
$$

Proof Note first that we always have $1 / r_{1} \leq x_{3}$ since

$$
D_{1}\left(1 / r_{1}\right)=\left(1-1 / r_{2}\right)^{2} / r_{1}^{2} \geq 0 \text {. }
$$

In case $\mathrm{I}$, note that if $r_{2} \leq 1,1 / r_{1}$ is a removable singularity for the function $P(x, 1)$. If $r_{2}>1$, then $x^{*}<1 / r_{1} \leq x_{3}$ since

$$
Q_{y}\left(1 / r_{1}\right)=v_{1} \lambda\left(1 / r_{2}-p-(1-p) / r_{1}\right)<0
$$

This implies that $x^{*}$ is the singularity with the smallest modulus. The residue of the function $P(x, 0)$ at point $x^{*}$ is equal to

$$
(1-\rho) \frac{\nu_{2} y^{*}\left(p v_{1} y^{*}-\lambda\left(x^{*}\right)^{2}\right)}{\left.(1-p) x^{*} \mathcal{Q}_{x}^{\prime}\left(y^{*}\right) \frac{\partial Y^{*}}{\partial x}\right|_{x=x^{*}}} .
$$

Since $h_{1}\left(x, Y^{*}(x)\right)=0$, we deduce that

$$
\left.\frac{\partial Y^{*}}{\partial x}\right|_{x=x^{*}}=-\frac{\frac{\partial h_{1}}{\partial x}\left(x^{*}, y^{*}\right)}{\frac{\partial h_{1}}{\partial y}\left(x^{*}, y^{*}\right)}=\frac{\left(y^{*}\right)^{2}\left(p v_{1} y^{*}-\lambda\left(x^{*}\right)^{2}\right)}{x^{*}\left(p v_{1}\left(y^{*}\right)^{2}-(1-p) \nu_{2} x^{*}\right)} .
$$

A direct application of Darboux's method then yields (23).

In case II, the point $r_{1} \leq 1$ is the pole with the smallest modulus for the function $P(x, 1)$ and Darboux's method yields $(24)$.

In case III, the function $P(x, 1)$ has no singularities in the disk $D\left(0, x_{3}\right)$ with center 0 and radius $x_{3}$. The function $P(x, 0)$ can then be represented, for $|x|<x_{3}$, as

$$
P(x, 0)=\frac{1}{2 i \pi} \int_{C\left(x_{3}\right)} \frac{g_{x}(z)}{z-x} d z
$$


where $C\left(x_{3}\right)$ is the circle with center 0 and radius $x_{3}$. By using (19), we have

$$
P(x, 1)=v_{1}(1-p) \frac{P(x, 0)-P\left(1 / r_{1}, 0\right)}{\lambda x-p v_{1}}=\frac{1}{2 i \pi} \int_{C\left(x_{3}\right)} \frac{h_{x}(z)}{z-x} d z,
$$

where

$$
h_{x}(z)=v_{1}(1-p) \frac{g_{x}(z)}{\lambda z-p v_{1}} .
$$

As shown in Sect. 3, the point $x_{*}$ may be a pole of the function $h_{x}$. Let $\operatorname{Res}\left(h_{x} ; x_{*}\right)$ denote the residue of the function $h_{x}$ at $x_{*}$. By deforming the integration contour so as to encompass the segment $\left[x_{3}, x_{4}\right]$, and since $\left|h_{x}(z)\right|<K_{x} /|z|$ for some constant $K_{x}>0$ when $|z| \rightarrow \infty$, we deduce that

$$
P(x, 1)=\frac{1}{2 i \pi} \int_{x_{3}}^{x_{4}} \frac{h_{x}(z+0 i)-h_{x}(z-0 i)}{z-x} d z+\frac{\operatorname{Res}\left(h_{x} ; x_{*}\right)}{x-x_{*}}
$$

and then

$$
\begin{aligned}
P(x, 1)= & \frac{-1}{\pi} \int_{x_{3}}^{x_{4}} \frac{(1-\rho) v_{1} v_{2}}{\xi\left(\lambda \xi-p v_{1}\right)(\xi-x)} \Im\left(\frac{Y^{*}(\xi)\left(\lambda \xi^{2}-p v_{1} Y^{*}(\xi)\right)}{\mathcal{Q}_{x}\left(Y^{*}(\xi)\right)}\right) d \xi \\
& +\frac{\operatorname{Res}\left(h_{x} ; x_{*}\right)}{x-x_{*}} .
\end{aligned}
$$

We have

$$
\Im\left(\frac{Y^{*}(\xi)\left(\lambda \xi^{2}-p v_{1} Y^{*}(\xi)\right)}{\mathcal{Q}_{x}\left(Y^{*}(\xi)\right)}\right)=\frac{\Im\left(Y^{*}(\xi)\left(\lambda \xi^{2}-p v_{1} Y^{*}(\xi)\right) \mathcal{Q}_{x}\left(\overline{Y^{*}(\xi)}\right)\right)}{\mathcal{Q}_{x}\left(Y^{*}(\xi)\right) \mathcal{Q}_{x}\left(\overline{Y^{*}(\xi)}\right)} .
$$

When $\xi \in\left[x_{3}, x_{4}\right]$, the relation

$$
\overline{Y^{*}}(\xi)=Y_{*}(\xi)
$$

holds and tedious computations show that $\mathcal{Q}_{x}\left(Y^{*}(\xi)\right) \mathcal{Q}_{x}\left(Y_{*}(\xi)\right)$ is a quadratic polynomial in $\xi$. In particular, we get

$\mathcal{Q}_{x}\left(Y^{*}(\xi)\right) \mathcal{Q}_{x}\left(Y_{*}(\xi)\right)=\left(\lambda \nu_{1}\right)^{2}\left(Y^{*}(\xi)-y^{*}\right)\left(Y^{*}(\xi)-y_{*}\right)\left(Y_{*}(\xi)-y^{*}\right)\left(Y_{*}(\xi)-y_{*}\right)$.

By definition, we know that the above quantity vanishes for $x$ equal to $x^{*}$ or $x_{*}$. More precisely, in case III, we have $Y_{*}\left(x^{*}\right)=y^{*}$. In addition, $Y_{*}\left(x_{*}\right)$ or $Y^{*}\left(x_{*}\right)$ is equal to $y_{*}$. Finally, we note that if $x$ is such that $h_{1}(x, y)=0$ then $p v_{1} y /(\lambda x)$ is also such that $h_{1}(x, y)=0$. This implies that the four roots of the polynomial $\mathcal{Q}_{x}\left(Y^{*}(\xi)\right) \mathcal{Q}_{x}\left(Y_{*}(\xi)\right)$ are $x_{*}, x^{*}, p v_{1} y^{*} /\left(\lambda x^{*}\right)$ and $p v_{1} y_{*} /\left(\lambda x_{*}\right)$. Hence,

$$
\begin{aligned}
\mathcal{Q}_{x}\left(Y^{*}(\xi)\right) \mathcal{Q}_{x}\left(Y_{*}(\xi)\right) & =-\frac{\lambda^{3} v_{2}^{2}}{p^{2} v_{1}}\left(\xi-x_{*}\right)\left(\xi-x^{*}\right)\left(\xi-\frac{p v_{1} y^{*}}{\lambda x^{*}}\right)\left(\xi-\frac{p v_{1} y_{*}}{\lambda x_{*}}\right) \\
& =-\frac{\lambda v_{2}^{2}}{p^{2} v_{1}} \mathcal{Q}_{y}(\xi) \mathcal{Q}_{y}^{*}(\xi),
\end{aligned}
$$


where the polynomial $\mathcal{Q}_{y}^{*}(x)$ is defined by (5).

Moreover, we have

$$
\begin{aligned}
& \Im\left(Y^{*}(\xi)\left(p v_{1} Y^{*}(\xi)-\lambda \xi^{2}\right) \mathcal{Q}_{x}\left(\overline{Y^{*}(\xi)}\right)\right) \\
& \quad=\frac{\lambda}{2 p \nu_{1}}\left(-\frac{\lambda^{2} v_{2}(1-p)}{p} \xi^{3}+v_{2}^{2}\left(p \lambda+v_{1}\right) \xi-2 v_{2}^{2} \lambda \xi^{2}\right) \sqrt{-D_{1}(\xi)} .
\end{aligned}
$$

It follows that

$$
P(x, 1)=\frac{1}{\pi} \int_{x_{3}}^{x_{4}} \frac{H_{x}(\xi)}{\xi-x} d \xi+\frac{\operatorname{Res}\left(h_{x} ; x_{*}\right)}{x-x_{*}},
$$

where

$$
H_{x}(\xi)=\frac{(1-\rho) \nu_{1}}{p \nu_{1}-\lambda \xi} \frac{\lambda^{2}(1-p) \xi^{2}+2 p \lambda \nu_{2} \xi-p v_{2}\left(p \lambda+v_{1}\right)}{2 \mathcal{Q}_{y}(\xi) \mathcal{Q}_{y}^{*}(\xi)} \sqrt{-D_{1}(\xi)}
$$

Then

$$
\mathbb{P}\left(N_{1}=n\right)=\frac{1}{\pi} \int_{x_{3}}^{x_{4}} \frac{H_{x}(\xi)}{\xi} e^{-n \log \xi} d \xi-\frac{\operatorname{Res}\left(h_{x} ; x_{*}\right)}{\left(x_{*}\right)^{n+1}} .
$$

In the neighborhood of $x_{3}$, we have

$$
-\log \xi=-\log x_{3}-\frac{1}{x_{3}}\left(\xi-x_{3}\right)+o\left(\xi-x_{3}\right)
$$

and

$$
\frac{H_{x}(\xi)}{\pi \xi}=k_{3}^{(1)} \sqrt{\xi-x_{3}}+o\left(\sqrt{\xi-x_{3}}\right)
$$

where

$$
k_{3}^{(1)}=\frac{(1-\rho) \nu_{1}}{2 \pi\left(p v_{1}-\lambda x_{3}\right)} \frac{\lambda^{2}(1-p) x_{3}^{2}+2 p v_{2} \lambda x_{3}-p \nu_{2}\left(p \lambda+v_{1}\right)}{x_{3} \mathcal{Q}_{y}\left(x_{3}\right) \mathcal{Q}_{y}^{*}\left(x_{3}\right)} \tau_{x},
$$

with $\tau_{x}$ as in (27). A direct application of Laplace's method [6, 8] then yields

$$
\mathbb{P}\left(N_{1}=n\right) \sim k_{3}^{(1)} \Gamma(3 / 2) \frac{1}{n^{3 / 2}}\left(\frac{1}{x_{3}}\right)^{n-\frac{3}{2}}
$$

when $n \rightarrow \infty$. Since $\Gamma(3 / 2)=\sqrt{\pi} / 2$, (25) follows.

In case IV, we have for $\xi$ in the neighborhood of $x_{3}$

$$
\mathcal{Q}_{y}(\xi)=\mathcal{Q}_{y}^{\prime}\left(x_{3}\right)\left(\xi-x_{3}\right)+o\left(\xi-x_{3}\right)
$$

and then

$$
\frac{H_{x}(\xi)}{2 \pi \xi}=k_{4}^{(1)}\left(\xi-x_{3}\right)^{-1 / 2}+o\left(\left(\xi-x_{3}\right)^{-1 / 2}\right),
$$


where

$$
k_{4}^{(1)}=\frac{(1-\rho) \nu_{1}}{2 \pi\left(p v_{1}-\lambda x_{3}\right)} \frac{\lambda^{2}(1-p) x_{3}^{2}+2 p \nu_{2} \lambda x_{3}-p \nu_{2}\left(p \lambda+v_{1}\right)}{x_{3} \mathcal{Q}_{y}^{\prime}\left(x_{3}\right) \mathcal{Q}_{y}^{*}\left(x_{3}\right)} \tau_{x} .
$$

Laplace's method then yields

$$
\mathbb{P}\left(N_{1}=n\right) \sim k_{4}^{(1)} \Gamma(1 / 2) \frac{1}{n^{1 / 2}}\left(\frac{1}{x_{3}}\right)^{n-\frac{1}{2}}
$$

and by using the fact that $\Gamma(1 / 2)=\sqrt{\pi}$, (26) follows.

Remark When we set $p=0$ we give full priority to queue 2 and the functional equation greatly simplifies due to $h_{3}(x, y)=0$. Then, for $\zeta(x)=v_{2} /\left(\lambda+v_{2}-\lambda x\right)$, we see that $h_{1}(x, \zeta(x))=0$ and hence

$$
\begin{aligned}
P(x, 0) & =-\frac{h_{4}(x, \zeta(x)) P(0,0)}{h_{2}(x, \zeta(x))}=\frac{\left(\nu_{1} v_{2}-\lambda v_{1} x\right)(1-\rho)}{\mathcal{Q}_{y}(x)} \\
& =\frac{\left(v_{1} v_{2}-\lambda v_{1} x\right)(1-\rho)}{\lambda^{2}\left(x-x_{*}\right)\left(x-x^{*}\right)}=\frac{c_{1}}{x-x_{*}}+\frac{c_{2}}{x-x^{*}},
\end{aligned}
$$

with

$$
c_{1}=\frac{\left(v_{1} v_{2}-\lambda v_{1} x_{*}\right)(1-\rho)}{\lambda^{2}\left(x_{*}-x^{*}\right)}, \quad c_{2}=\frac{\left(v_{1} v_{2}-\lambda v_{1} x^{*}\right)(1-\rho)}{\lambda^{2}\left(x_{*}-x^{*}\right)} .
$$

This gives

$$
P(x, 1)=\frac{\nu_{1}}{\lambda x}\left[\frac{c_{1}}{x-x_{*}}+\frac{c_{2}}{x-x^{*}}-(1-\rho)\right]
$$

and

$$
\mathbb{P}\left(N_{1}=n\right) \sim \frac{v_{1}^{2} \lambda x^{*}-v_{1}^{2} v_{2}}{\lambda^{3}\left(x^{*}-x_{*}\right)\left(x^{*}\right)^{2}}(1-\rho)\left(\frac{1}{x^{*}}\right)^{n} .
$$

Note that this agrees with regime I in Proposition 5 if

$$
\frac{v_{1}\left(\lambda x^{*}-v_{2}\right)}{\lambda^{2}\left(x^{*}-x_{*}\right) x^{*}}=\frac{v_{2}^{2}}{v_{2}^{2}+\lambda v_{1}\left(y_{*}\right)^{2}},
$$

which can indeed be shown to be true.

For the second queue, we first note by using Lemma 6 that the point $r_{1} / r_{2}$ is always a removable singularity for the function $P(1, y)$.

Proposition 6 For given system parameters $\lambda, v_{1}, v_{2}$ and $p$, the exact asymptotics for $\mathbb{P}\left(N_{2}=n\right)$ as $n \rightarrow \infty$ are: 
I If $x^{*}=X^{*}\left(y^{*}\right)$ and $y^{*}<y_{3}$, which can occur only when $r_{1} \leq 1$, then

$$
\mathbb{P}\left(N_{2}=n\right) \sim \kappa_{1}^{(2)}\left(\frac{1}{y^{*}}\right)^{n} .
$$

II If $x^{*} \neq X^{*}\left(y^{*}\right)$, then

$$
\mathbb{P}\left(N_{2}=n\right) \sim \kappa_{2}^{(2)} \frac{1}{n \sqrt{n}}\left(\frac{1}{y_{3}}\right)^{n}
$$

III If $x^{*}=X^{*}\left(y^{*}\right)$ and $y^{*}=y_{3}$,

$$
\mathbb{P}\left(N_{2}=n\right) \sim \kappa_{3}^{(2)} \frac{1}{\sqrt{n}}\left(\frac{1}{y^{*}}\right)^{n} .
$$

with $y_{3}$ the third largest real root of $D_{2}(y)$ in (3), and $X^{*}(y), y^{*}, x^{*}$ as in (5), (13), (16), respectively. Further, $r_{i}=\lambda /\left(p v_{i}\right), \rho=1-\lambda / \nu_{1}-\lambda / \nu_{2}$ and

$\kappa_{1}^{(2)}=\frac{(1-\rho) \lambda\left(v_{1} y^{*}+v_{2}\right)\left(p v_{1} y^{*}-\lambda\left(x^{*}\right)^{2}\right)}{\left((1-p) v_{2}-p v_{1} y^{*}\right) x^{*} \mathcal{Q}_{y}^{\prime}\left(x^{*}\right)}$,

$\kappa_{2}^{(2)}=\frac{(1-\rho)\left(\nu_{2}+v_{1} y_{3}\right)\left(\lambda p\left(p v_{2}+(1-p) v_{1}\right) y_{3}^{2}+2 \lambda p(1-p) \nu_{2} y_{3}-(1-p) v_{2}^{2}\right)}{2 \sqrt{\pi} p^{2}\left(p v_{1} y_{3}-(1-p) \nu_{2}\right) \mathcal{Q}_{x}\left(y_{3}\right) \mathcal{Q}_{x}^{*}\left(y_{3}\right)} \sqrt{y_{3}} \tau_{y}$,

$\kappa_{3}^{(2)}=\frac{(1-\rho)\left(v_{2}+v_{1} y_{3}\right)\left(\lambda p\left(p v_{2}+(1-p) v_{1}\right) y_{3}^{2}+2 \lambda p(1-p) \nu_{2} y_{3}-(1-p) v_{2}^{2}\right)}{\sqrt{\pi} \sqrt{y_{3}} p^{2}\left(p v_{1} y_{3}-(1-p) \nu_{2}\right) \mathcal{Q}_{x}^{\prime}\left(y_{3}\right) \mathcal{Q}_{x}^{*}\left(y_{3}\right)} \tau_{y}$,

with $\tau_{y}=\sqrt{p v_{1}\left(y_{3}-y_{1}\right)\left(y_{3}-y_{2}\right) / \lambda}$,

$$
\mathcal{Q}_{x}^{*}(y)=\left(y-\frac{(1-p) v_{2} x^{*}}{p v_{1} y^{*}}\right)\left(y-\frac{(1-p) v_{2} x_{*}}{p v_{1} y_{*}}\right),
$$

and $\mathcal{Q}_{x}(y), \mathcal{Q}_{y}(x)$ as in (11), (15), respectively.

Proof In case I, $y^{*}$ is the pole with the smallest modulus for the function $P(1, y)$ and a direct application of Darboux's method yields

$$
\mathbb{P}\left(N_{2}=n\right) \sim \frac{(1-\rho) \lambda}{(1-p) \nu_{2}-p v_{1} y^{*}} \frac{(1-p) \nu_{2} x^{*}-p v_{1}\left(y^{*}\right)^{2}}{\left.\left(y^{*}\right)^{2} \mathcal{Q}_{y}^{\prime}\left(x^{*}\right) \frac{\partial X^{*}}{\partial y}\right|_{y=y^{*}}}\left(\frac{1}{y^{*}}\right)^{n}
$$

and (29) follows.

In case II, the function $P(1, y)$ is analytic in the disk with center 0 and radius $y_{3}$ and we have

$$
P(0, y)=\frac{1}{2 i \pi} \int_{C\left(y_{3}\right)} \frac{g_{y}(z)}{z-y} d z,
$$

where $C\left(y_{3}\right)$ is the circle with center 0 and radius $y_{3}$. By using (22), we have

$$
P(1, y)=1-\rho+\frac{\left(v_{1} y+v_{2}\right) p\left(P\left(0, r_{1} / r_{2}\right)-P(0, y)\right)}{(1-p) \nu_{2}-p v_{1} y}
$$




$$
=1-\rho+\frac{1}{2 i \pi} \int_{C\left(y_{3}\right)} \frac{h_{y}(z)}{z-x} d z
$$

where

$$
h_{y}(z)=\frac{p\left(v_{2}+v_{1} z\right) g_{y}(z)}{p v_{1} z-(1-p) v_{2}} .
$$

By deforming the integration contour along the segment $\left[y_{3}, \infty\right)$, and since the function $h_{y}(z)$ is such that $\left|h_{y}(z)\right|<K_{y} /|z|$ for some constant $K_{y}>0$ when $|z| \rightarrow$ $\infty$, we deduce that

$$
P(1, y)=(1-\rho)+\frac{1}{2 i \pi} \int_{y_{3}}^{\infty} \frac{h_{y}(z+0 i)-h_{y}(z-0 i)}{z-y} d z
$$

and then

$$
\begin{aligned}
& P(1, y) \\
& \quad=(1-\rho)+\frac{-1}{\pi} \int_{y_{3}}^{\infty} \frac{(1-\rho) \lambda\left(\nu_{2}+v_{1} z\right)}{z\left(p v_{1} z-(1-p) \nu_{2}\right)} \Im\left(\frac{\left(p v_{1} y^{2}-(1-p) \nu_{2} X^{*}(y)\right)}{\mathcal{Q}_{y}\left(X^{*}(y)\right)}\right) d z .
\end{aligned}
$$

There holds

$$
\Im\left(\frac{\left(p v_{1} y^{2}-(1-p) \nu_{2} X^{*}(y)\right)}{\mathcal{Q}_{y}\left(X^{*}(y)\right)}\right)=\frac{\Im\left(\left(p v_{1} y^{2}-(1-p) \nu_{2} X^{*}(y)\right) \mathcal{Q}_{y}\left(\overline{X^{*}(y)}\right)\right)}{\mathcal{Q}_{y}\left(X^{*}(y)\right) \mathcal{Q}_{y}\left(\overline{X^{*}(y)}\right)} .
$$

When $z \in\left[y_{3}, \infty\right)$, we have

$$
\overline{X^{*}}(z)=X_{*}(z) \text {. }
$$

It is easily checked that the function $z \rightarrow z^{2} \mathcal{Q}_{y}\left(X^{*}(z)\right) \mathcal{Q}_{y}\left(X_{*}(z)\right)$ is a quadratic polynomial in $z$. By definition, we know that this polynomial vanishes for $y$ equal to $y^{*}$ or $y_{*}$. More precisely, in case II, we have $X_{*}\left(y^{*}\right)=x^{*}$. In addition, $X_{*}\left(y_{*}\right)$ or $X^{*}\left(y_{*}\right)$ is equal to $x_{*}$. If $y$ is such that $h_{1}(x, y)=0$ then $(1-p) v_{2} x /\left(p v_{1} y\right)$ is also such that $h_{1}(x, y)=0$. This implies that the four roots of the polynomial $z^{2} \mathcal{Q}_{y}\left(X^{*}(z)\right) \mathcal{Q}_{y}\left(X_{*}(z)\right)$ are $y_{*}, y^{*},(1-p) v_{2} x^{*} /\left(p v_{1} y^{*}\right)$ and $(1-p) v_{2} x_{*} /\left(p v_{1} y_{*}\right)$. Hence,

$$
\begin{aligned}
& z^{2} \mathcal{Q}_{y}\left(X^{*}(z)\right) \mathcal{Q}_{y}\left(X_{*}(z)\right) \\
& \quad=\lambda^{2} p^{2} v_{1}^{2}\left(z-y_{*}\right)\left(z-y^{*}\right)\left(z-\frac{(1-p) \nu_{2} x^{*}}{p v_{1} y^{*}}\right)\left(z-\frac{(1-p) \nu_{2} x_{*}}{p v_{1} y_{*}}\right)
\end{aligned}
$$

and then

$$
z^{2} \mathcal{Q}_{y}\left(X^{*}(z)\right) \mathcal{Q}_{y}\left(X_{*}(z)\right)=\lambda v_{1} p^{2} \mathcal{Q}_{x}(z) \mathcal{Q}_{x}^{*}(z),
$$

where the polynomial $\mathcal{Q}_{x}^{*}(z)$ as defined in (32). 
Moreover, we have in the neighborhood of $y_{3}$

$$
\begin{aligned}
& \Im\left(\left(p v_{1} y^{2}-(1-p) \nu_{2} X^{*}(y)\right) \mathcal{Q}_{y}\left(\overline{X^{*}(y)}\right)\right) \\
& \quad=-\frac{\nu_{1}}{2 y}\left(\lambda p\left(p v_{2}+(1-p) \nu_{1}\right) y^{2}+2 \lambda p(1-p) \nu_{2} y-(1-p) v_{2}^{2}\right) \sqrt{-D_{2}(y)} .
\end{aligned}
$$

It follows that

$$
P(1, y)=(1-\rho)+\frac{1}{\pi} \int_{y_{3}}^{\infty} \frac{H_{y}(z)}{z-x} d z,
$$

where the function $H_{y}(z)$ is defined for $z$ in the neighborhood of $y_{3}$ by

$$
\begin{aligned}
H_{y}(z)= & \frac{(1-\rho)\left(v_{2}+v_{1} z\right) \sqrt{-D_{2}(z)}}{2 p^{2}\left(p v_{1} z-(1-p) v_{2}\right) \mathcal{Q}_{x}(z) \mathcal{Q}_{x}^{*}(z)} \\
& \times\left(\lambda p\left(p v_{2}+(1-p) v_{1}\right) z^{2}+2 \lambda p(1-p) v_{2} z-(1-p) v_{2}^{2}\right)
\end{aligned}
$$

Then, for $n \geq 1$,

$$
\mathbb{P}\left(N_{2}=n\right)=\frac{1}{\pi} \int_{y_{3}}^{\infty} \frac{H_{y}(z)}{z} e^{-n \log z} d z .
$$

In the neighborhood of $y_{3}$, we have

$$
-\log z=-\log y_{3}-\frac{1}{y_{3}}\left(z-y_{3}\right)+o\left(z-y_{3}\right)
$$

and

$$
\frac{H_{y}(z)}{\pi z}=k_{2}^{(2)} \sqrt{z-y_{3}}+o\left(\sqrt{z-y_{3}}\right),
$$

where

$$
\begin{aligned}
k_{2}^{(2)}= & \frac{(1-\rho)\left(v_{2}+v_{1} y_{3}\right)\left(\lambda p\left(p v_{2}+(1-p) v_{1}\right) y_{3}^{2}+2 \lambda p(1-p) v_{2} y_{3}-(1-p) v_{2}^{2}\right)}{2 \pi y_{3} p^{2}\left(p v_{1} y_{3}-(1-p) \nu_{2}\right) \mathcal{Q}_{x}\left(y_{3}\right) \mathcal{Q}_{x}^{*}\left(y_{3}\right)} \\
& \times \sqrt{4 p v_{1}\left(y_{3}-y_{1}\right)\left(y_{3}-y_{2}\right) / \lambda} .
\end{aligned}
$$

A direct application of Laplace's method then yields

$$
\mathbb{P}\left(N_{2}=n\right) \sim k_{2}^{(2)} \Gamma(3 / 2) \frac{1}{n^{3 / 2}}\left(\frac{1}{y_{3}}\right)^{n-\frac{3}{2}}
$$

when $n \rightarrow \infty$. Since $\Gamma(3 / 2)=\sqrt{\pi} / 2$, (30) follows.

In case III, we have for $z$ in the neighborhood of $y_{3}$

$$
\mathcal{Q}_{x}(z)=\mathcal{Q}_{x}^{\prime}\left(y_{3}\right)\left(z-y_{3}\right)+o\left(\left(z-y_{3}\right)\right)
$$

and then

$$
\frac{H_{y}(z)}{2 \pi z}=k_{3}^{(2)}\left(z-y_{3}\right)^{-1 / 2}+o\left(\left(z-y_{3}\right)^{-1 / 2}\right),
$$


where

$$
\begin{aligned}
k_{3}^{(2)}= & \frac{(1-\rho)\left(v_{2}+v_{1} y_{3}\right)\left(\lambda p\left(p v_{2}+(1-p) \nu_{1}\right) y_{3}^{2}+2 \lambda p(1-p) \nu_{2} y_{3}-(1-p) v_{2}^{2}\right)}{2 \pi y_{3} p^{2}\left(p v_{1} y_{3}-(1-p) \nu_{2}\right) \mathcal{Q}_{x}^{\prime}\left(y_{3}\right) \mathcal{Q}_{x}^{*}\left(y_{3}\right)} \\
& \times \sqrt{4 p v_{1}\left(y_{3}-y_{1}\right)\left(y_{3}-y_{2}\right) / \lambda} .
\end{aligned}
$$

Laplace's method then yields

$$
\mathbb{P}\left(N_{2}=n\right) \sim k_{3}^{(2)} \Gamma(1 / 2) \frac{1}{n^{1 / 2}}\left(\frac{1}{y_{3}}\right)^{n-\frac{1}{2}}
$$

and with $\Gamma(1 / 2)=\sqrt{\pi}(31)$ follows.

\subsection{Numerical examples}

We shall now compare the asymptotic estimates in Propositions 5 and 6 against results obtained by numerical calculations. Truncating the state space by bounding one of the queue lengths leads to a Markov process on an infinite strip, better known as a Quasi-Birth-Death (QBD) process. For these processes, fast numerical algorithms are available (see [26]). All numerical results presented were obtained by imposing an upper bound on the second queue of 500 (so that the truncation effect should be negligible).

For a first scenario we take $\lambda=1.5, \rho_{1}=.4$ and $\rho_{2}=.3$. Figure 5 compares $X^{*}\left(y^{*}\right)$ with $x^{*}$ and $Y^{*}\left(x^{*}\right)$ with $y^{*}$, when $p$ varies. For example, we see that for $p<.6, Y^{*}\left(x^{*}\right)=y^{*}$. For $p=.5$, we have regime (23) for queue 1 and regime (30) for queue 2. Results for this case are presented in Table 1. Note that (23) converges fast to the true (numerical) value. The convergence of the branch point asymptotics (30) seems slower, in particular the convergence of the last column in Table 1 to the value $\kappa_{2}^{(2)}=20.7454$. In order to demonstrate that $\kappa_{2}^{(2)}$ is indeed the leading constant, we

Fig. 5 Comparisons of $x^{*}$, $X^{*}\left(y^{*}\right)$ and $y^{*}, Y^{*}\left(x^{*}\right)$ when $p$ varies. Here, $\lambda=1.5, \rho_{1}=.4$, $\rho_{2}=.3$ and $x^{*}=1.5890$, $y^{*}=1.2146$

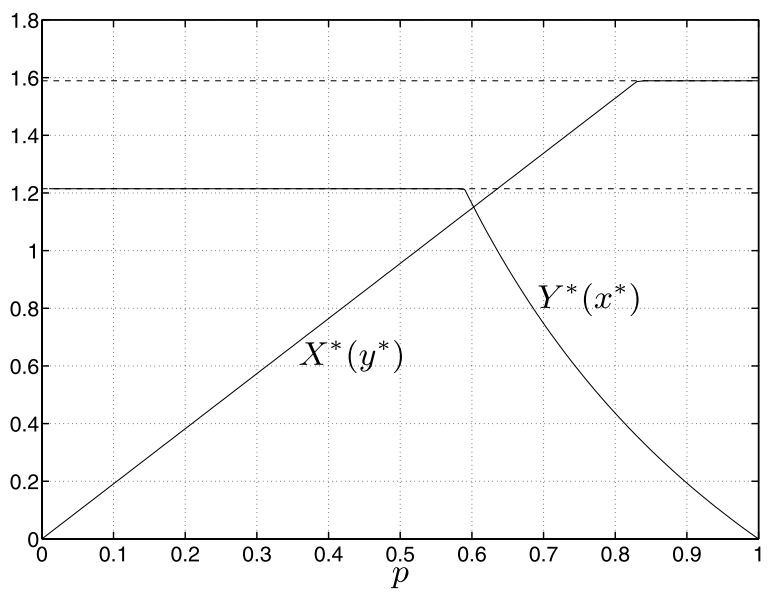


Table 1 Illustration of (23) and (30) for $\lambda=1.5, \rho_{1}=.4, \rho_{2}=.3, p=.5$. In this case $x^{*}=1.5890$, $X^{*}\left(y^{*}\right)=0.9555, y^{*}=Y^{*}\left(x^{*}\right)=1.2146$. We find that $\kappa_{2}^{(2)}=20.7454$

\begin{tabular}{rlllll}
\hline \multicolumn{1}{l}{$n$} & $\mathbb{P}\left(N_{1}=n\right)$ & $\kappa_{1}^{(1)}\left(x^{*}\right)^{-n}$ & $\mathbb{P}\left(N_{2}=n\right)$ & $n^{-3 / 2}\left(y_{3}\right)^{-n}$ & $\frac{\mathbb{P}\left(N_{2}=n\right)}{n^{-3 / 2}\left(y_{3}\right)^{-n}}$ \\
\hline 5 & $2.8301 \mathrm{e}-002$ & $2.4891 \mathrm{e}-002$ & $1.0567 \mathrm{e}-002$ & $5.1414 \mathrm{e}-003$ & $2.0553 \mathrm{e}+000$ \\
10 & $2.5852 \mathrm{e}-003$ & $2.4569 \mathrm{e}-003$ & $3.6384 \mathrm{e}-004$ & $1.0449 \mathrm{e}-004$ & $3.4821 \mathrm{e}+000$ \\
15 & $2.4842 \mathrm{e}-004$ & $2.4252 \mathrm{e}-004$ & $1.5032 \mathrm{e}-005$ & $3.2693 \mathrm{e}-006$ & $4.5978 \mathrm{e}+000$ \\
20 & $2.4237 \mathrm{e}-005$ & $2.3938 \mathrm{e}-005$ & $6.7391 \mathrm{e}-007$ & $1.2206 \mathrm{e}-007$ & $5.5210 \mathrm{e}+000$ \\
50 & $2.2151 \mathrm{e}-011$ & $2.2140 \mathrm{e}-011$ & $1.0132 \mathrm{e}-014$ & $1.1140 \mathrm{e}-015$ & $9.0958 \mathrm{e}+000$ \\
100 & $1.9438 \mathrm{e}-021$ & $1.9438 \mathrm{e}-021$ & $1.8829 \mathrm{e}-027$ & $1.5511 \mathrm{e}-028$ & $1.2139 \mathrm{e}+001$ \\
200 & $1.4983 \mathrm{e}-041$ & $1.4983 \mathrm{e}-041$ & $1.2762 \mathrm{e}-052$ & $8.5067 \mathrm{e}-054$ & $1.5002 \mathrm{e}+001$ \\
300 & $1.1549 \mathrm{e}-061$ & $1.1549 \mathrm{e}-061$ & $1.1804 \mathrm{e}-077$ & $7.1825 \mathrm{e}-079$ & $1.6434 \mathrm{e}+001$ \\
\hline
\end{tabular}

Table 2 Comparison of (33) and (30) for $\lambda=1.5, \rho_{1}=.4$, $\rho_{2}=.3, p=.5$ and $\kappa_{2}^{(2)}=20.7454$

\begin{tabular}{llll}
\hline$n$ & $(33)$ & $n^{-3 / 2}\left(y_{3}\right)^{-n}$ & $\frac{\mathbb{P}\left(N_{2}=n\right)}{n^{-3 / 2}\left(y_{3}\right)^{-n}}$ \\
\hline $10^{2}$ & $1.8301 \mathrm{e}-27$ & $1.5509 \mathrm{e}-28$ & $1.1801 \mathrm{e}+1$ \\
$10^{3}$ & $4.8227 \mathrm{e}-252$ & $2.5453 \mathrm{e}-253$ & $1.8947 \mathrm{e}+1$ \\
$10^{4}$ & $2.3446 \mathrm{e}-2486$ & $1.1415 \mathrm{e}-2487$ & $2.0540 \mathrm{e}+1$ \\
$10^{5}$ & $2.4607 \mathrm{e}-24816$ & $1.1873 \mathrm{e}-24817$ & $2.0725 \mathrm{e}+1$ \\
$10^{6}$ & $1.1550 \mathrm{e}-248102$ & $5.5682 \mathrm{e}-248104$ & $2.0743 \mathrm{e}+1$ \\
$10^{7}$ & $1.8797 \mathrm{e}-2480952$ & $9.0611 \mathrm{e}-2480954$ & $2.0745 \mathrm{e}+1$ \\
\hline
\end{tabular}

Table 3 Illustration of (25) and (30) for $\lambda=1.5, \rho_{1}=.4, \rho_{2}=.3, p=.65$. In this case $x^{*}=1.5890$, $X^{*}\left(y^{*}\right)=1.2421, y^{*}=1.2146$ and $Y^{*}\left(x^{*}\right)=0.9392$. We find that $\kappa_{3}^{(1)}=81.6727$ and $\kappa_{2}^{(2)}=3.7799$

\begin{tabular}{rllllll}
\hline$n$ & $\mathbb{P}\left(N_{1}=n\right)$ & $n^{-3 / 2}\left(x_{3}\right)^{-n}$ & $\frac{\mathbb{P}\left(N_{1}=n\right)}{n^{-3 / 2}\left(x_{3}\right)^{-n}}$ & $\mathbb{P}\left(N_{2}=n\right)$ & $n^{-3 / 2}\left(y_{3}\right)^{-n}$ & $\frac{\mathbb{P}\left(N_{2}=n\right)}{n^{-3 / 2}\left(y_{3}\right)^{-n}}$ \\
\hline 5 & $2.0854 \mathrm{e}-002$ & $7.4520 \mathrm{e}-003$ & $2.7985 \mathrm{e}+000$ & $2.6154 \mathrm{e}-002$ & $2.7103 \mathrm{e}-002$ & $9.6499 \mathrm{e}-001$ \\
10 & $1.2811 \mathrm{e}-003$ & $2.1951 \mathrm{e}-004$ & $5.8359 \mathrm{e}+000$ & $4.1746 \mathrm{e}-003$ & $2.9037 \mathrm{e}-003$ & $1.4377 \mathrm{e}+000$ \\
15 & $8.6268 \mathrm{e}-005$ & $9.9552 \mathrm{e}-006$ & $8.6656 \mathrm{e}+000$ & $8.3828 \mathrm{e}-004$ & $4.7896 \mathrm{e}-004$ & $1.7502 \mathrm{e}+000$ \\
20 & $6.0730 \mathrm{e}-006$ & $5.3873 \mathrm{e}-007$ & $1.1273 \mathrm{e}+001$ & $1.8669 \mathrm{e}-004$ & $9.4268 \mathrm{e}-005$ & $1.9804 \mathrm{e}+000$ \\
50 & $1.0651 \mathrm{e}-012$ & $4.5586 \mathrm{e}-014$ & $2.3364 \mathrm{e}+001$ & $4.9780 \mathrm{e}-008$ & $1.8464 \mathrm{e}-008$ & $2.6961 \mathrm{e}+000$ \\
100 & $9.3290 \mathrm{e}-024$ & $2.5976 \mathrm{e}-025$ & $3.5914 \mathrm{e}+001$ & $1.3411 \mathrm{e}-013$ & $4.2613 \mathrm{e}-014$ & $3.1472 \mathrm{e}+000$ \\
200 & $1.1821 \mathrm{e}-045$ & $2.3856 \mathrm{e}-047$ & $4.9552 \mathrm{e}+001$ & $2.2323 \mathrm{e}-024$ & $6.4202 \mathrm{e}-025$ & $3.4770 \mathrm{e}+000$ \\
300 & $1.9248 \mathrm{e}-067$ & $3.3732 \mathrm{e}-069$ & $5.7061 \mathrm{e}+001$ & $5.3829 \mathrm{e}-035$ & $1.4892 \mathrm{e}-035$ & $3.6146 \mathrm{e}+000$ \\
\hline
\end{tabular}

compare (30) against the integral representation (33) (omitting the residue term); see Table 2. Indeed, this confirms the correctness of $\kappa_{2}^{(2)}=20.7454$.

Results for $p=.65$ are presented in Table 3 in which case we have regime (25) for queue 1 and regime (30) for queue 2. Note again the slow convergence to the asymptotic constants $\kappa_{3}^{(1)}$ and $\kappa_{2}^{(2)}$. 
Table 4 Illustration of (24) and (29) for $\lambda=1, \rho_{1}=.1, \rho_{2}=.85, p=.3$. In this case $x^{*}=X^{*}\left(y^{*}\right)=$ $1.0581, y^{*}=1.0520$ and $Y^{*}\left(x^{*}\right)=0.2761$

\begin{tabular}{rlllll}
\hline \multicolumn{1}{l}{$n$} & $\mathbb{P}\left(N_{1}=n\right)$ & $\kappa_{2}^{(1)}\left(r_{1}\right)^{n}$ & rel. error & $\mathbb{P}\left(N_{2}=n\right)$ & $\kappa_{1}^{(2)}\left(y^{*}\right)^{-n}$ \\
\hline 5 & $2.5017 \mathrm{e}-003$ & $2.1008 \mathrm{e}-003$ & 1.1909 & $3.7599 \mathrm{e}-002$ & $3.7227 \mathrm{e}-002$ \\
10 & $1.0008 \mathrm{e}-005$ & $8.6452 \mathrm{e}-006$ & 1.1577 & $2.8912 \mathrm{e}-002$ & $2.8894 \mathrm{e}-002$ \\
15 & $4.0423 \mathrm{e}-008$ & $3.5577 \mathrm{e}-008$ & 1.1362 & $2.2428 \mathrm{e}-002$ & $2.2427 \mathrm{e}-002$ \\
20 & $1.6403 \mathrm{e}-010$ & $1.4641 \mathrm{e}-010$ & 1.1204 & $1.7407 \mathrm{e}-002$ & $1.7407 \mathrm{e}-002$ \\
50 & $7.6060 \mathrm{e}-025$ & $7.1109 \mathrm{e}-025$ & 1.0696 & $3.8058 \mathrm{e}-003$ & $3.8058 \mathrm{e}-003$ \\
100 & $1.0262 \mathrm{e}-048$ & $9.9052 \mathrm{e}-049$ & 1.0360 & $3.0199 \mathrm{e}-004$ & $3.0199 \mathrm{e}-004$ \\
200 & $1.9451 \mathrm{e}-096$ & $1.9219 \mathrm{e}-096$ & 1.0120 & $1.9014 \mathrm{e}-006$ & $1.9014 \mathrm{e}-006$ \\
300 & $3.7427 \mathrm{e}-144$ & $3.7292 \mathrm{e}-144$ & 1.0036 & $1.1972 \mathrm{e}-008$ & $1.1972 \mathrm{e}-008$ \\
\hline
\end{tabular}

Table 4 illustrates some results for $\lambda=1.5, \rho_{1}=.2, \rho_{2}=.4$ and $p=.4$, in which case we have regimes (24) and (29).

Open Access This article is distributed under the terms of the Creative Commons Attribution Noncommercial License which permits any noncommercial use, distribution, and reproduction in any medium, provided the original author(s) and source are credited.

\section{Appendix: The resultant of the polynomials $h_{1}$ and $h_{2}$}

Generally speaking, when we have two polynomials in two variables, say,

$$
\begin{aligned}
& f_{1}(x, y)=a_{0}(y)+a_{1}(y) x+\cdots+a_{n}(y) x^{n}, \\
& f_{2}(x, y)=b_{0}(y)+b_{1}(y) x+\cdots+b_{m}(y) x^{m},
\end{aligned}
$$

the resultant of the polynomials $f_{1}$ and $f_{2}$ with respect to $x$ is the determinant $\operatorname{Res}_{x}\left(f_{1}, f_{2}\right)$ of the matrix

$$
\left.\left\{\begin{array}{cccccc}
a_{n} & \cdots & a_{0} & 0 & \cdots & \cdots \\
0 & a_{n} & \cdots & a_{0} & 0 & \cdots \\
\cdots & \cdots & \cdots & \cdots & \cdots & \cdots \\
\cdots & \cdots & 0 & a_{n} & \cdots & a_{0} \\
b_{m} & \cdots & b_{0} & 0 & \cdots & \cdots \\
0 & b_{m} & \cdots & b_{0} & 0 & \cdots \\
\cdots & \cdots & \cdots & \cdots & \cdots & \cdots \\
\cdots & \cdots & 0 & b_{m} & \cdots & b_{0}
\end{array}\right)\right\} m \text { rows }
$$

which is a polynomial in $y$. The polynomials $f_{1}$ and $f_{2}$ have a common nontrivial root $\left(x_{0}, y_{0}\right)$ if and only if the resultant with respect to $x$ is 0 at $y_{0}$. This leads to the resolution of a polynomial equation. Note that by adding to the $(m+n)$ th column, the $i$ th column multiplied by $x^{m+n-i}$ for $0 \leq i<n+m, \operatorname{Res}_{x}\left(f_{1}, f_{2}\right)$ is equal to the 
determinant of the matrix

$$
\left(\begin{array}{cccccc}
a_{n} & \cdots & a_{0} & 0 & \cdots & x^{m-1} f_{1} \\
0 & a_{n} & \cdots & a_{0} & 0 & x^{m-2} f_{1} \\
\cdots & \cdots & \cdots & \cdots & \cdots & \cdots \\
\cdots & \cdots & 0 & a_{n} & \cdots & f_{1} \\
b_{m} & \cdots & b_{0} & 0 & \cdots & x^{n-1} f_{2} \\
0 & b_{m} & \cdots & b_{0} & 0 & x^{n-2} f_{2} \\
\cdots & \cdots & \cdots & \cdots & \cdots & \cdots \\
\cdots & \cdots & 0 & b_{m} & \cdots & f_{2}
\end{array}\right)
$$

which can written as $p(x, y) f_{1}(x, y)+q(x, y) f_{2}(x, y)$, where $p$ and $q$ are polynomials in variables $x$ and $y$.

\section{A.1 Resultant in $x$}

In the case of the polynomials $h_{1}(x, y)$ and $h_{2}(x, y)$, the resultant in $x$, denoted by $Q_{x}(y)$, is the determinant of the matrix

$$
\left(\begin{array}{ccc}
-\lambda y & a_{1}(y) & -p v_{1} y^{2} \\
b_{1}(y) & (1-p) v_{1} y^{2} & 0 \\
0 & b_{1}(y) & (1-p) v_{1} y^{2}
\end{array}\right)
$$

where $a_{1}(y)=\left(\lambda+p v_{1}+(1-p) \nu_{2}\right) y-(1-p) \nu_{2}$ and $b_{1}(y)=(1-p)\left(\left(v_{2}-v_{1}\right) y-\right.$ $\left.v_{2}\right)$. Straightforward computations show that

$$
Q_{x}(y)=-v_{1}(1-p)^{2} y^{2}(y-1) \mathcal{Q}_{x}(y),
$$

where

$$
\mathcal{Q}_{x}(y)=\lambda v_{1} y^{2}+v_{2}\left(v_{2}-v_{1}+\lambda\right) y-v_{2}^{2} .
$$

It is easily checked that the quadratic polynomial $\mathcal{Q}_{x}(y)$ has two roots of opposite sign, as mentioned in Sect. 3. The positive root is

$$
y^{*}=\frac{\nu_{2}}{2 \lambda v_{1}}\left(-\left(v_{2}-v_{1}+\lambda\right)+\sqrt{\left(\nu_{2}-v_{1}+\lambda\right)^{2}+4 \lambda v_{1}}\right)
$$

and the negative root is

$$
y_{*}=\frac{\nu_{2}}{2 \lambda \nu_{1}}\left(-\left(v_{2}-v_{1}+\lambda\right)-\sqrt{\left(v_{2}-v_{1}+\lambda\right)^{2}+4 \lambda v_{1}}\right)
$$

In addition, the value of this polynomial at the point 1 is equal to $\lambda\left(v_{1}+v_{2}\right)-v_{1} v_{2}=$ $v_{1} v_{2}\left(\rho_{1}+\rho_{2}-1\right)<0$, which implies that $y^{*}>1$. 


\section{A.2 Resultant in $y$}

The resultant in $y$ of the polynomials $h_{1}(x, y)$ and $h_{2}(x, y)$ is denoted by $Q_{y}(x)$ and is equal to the determinant of the matrix

$$
\left(\begin{array}{cccc}
-p v_{1} & \alpha_{1}(x) & -(1-p) v_{2} x & 0 \\
0 & -p v_{1} & \alpha_{1}(x) & -(1-p) v_{2} x \\
(1-p) v_{1} & (1-p)\left(v_{2}-v_{1}\right) x & -v_{2}(1-p) x & 0 \\
0 & (1-p) v_{1} & (1-p)\left(v_{2}-v_{1}\right) x & -v_{2}(1-p) x
\end{array}\right)
$$

where $\alpha_{1}(x)=x\left(\lambda+p v_{1}+(1-p) v_{2}-\lambda x\right)$. Straightforward computations show that

$$
Q_{y}(x)=-v_{2} v_{1}(1-p)^{2} x^{2}(x-1) \mathcal{Q}_{y}(x)
$$

with $\mathcal{Q}_{y}(x)=\lambda^{2} x^{2}-\left(\lambda+v_{1}+v_{2}\right) \lambda x+v_{1} v_{2}$. The quadratic polynomial $\mathcal{Q}_{y}(x)$ has two positive roots equal to

$$
x^{*}=\frac{\lambda+v_{1}+v_{2}-\sqrt{\left(\lambda+v_{1}+v_{2}\right)^{2}-4 v_{1} \nu_{2}}}{2 \lambda}
$$

and

$$
x_{*}=\frac{\lambda+v_{1}+v_{2}+\sqrt{\left(\lambda+v_{1}+v_{2}\right)^{2}-4 v_{1} v_{2}}}{2 \lambda}
$$

with $x^{*}<x_{*}$, and since $\mathcal{Q}_{y}(1)=v_{1} v_{2}\left(1-\rho_{1}-\rho_{2}\right)>0, x^{*}>1$.

\section{References}

1. Adan, I.J.B.F., Foley, R.D., McDonald, D.R.: Exact asymptotics for the stationary distribution of a Markov chain: a production model. Queueing Syst. 62, 311-344 (2009)

2. Borovkov, A.A., Mogul'skii, A.A.: Large deviations for Markov chains in the positive quadrant. Russ. Math. Surv. 56, 803-916 (2001)

3. Borst, S.C., Boxma, O.J., van Uitert, M.J.G.: Two coupled queues with heterogeneous traffic. In: Proceedings of ITC 17, pp. 1003-1014. North-Holland, Amsterdam (2001)

4. Bousquet-Mélou, M.: Walks in the quarter plane: Kreweras' algebraic model. Ann. Appl. Probab. 15, 1451-1491 (2005)

5. Cohen, J.W., Boxma, O.J.: Boundary Value Problems in Queueing System Analysis. North-Holland, Amsterdam (1983)

6. De Bruijn, N.G.: Asymptotic Methods in Analysis. Dover, New York (1981)

7. Denteneer, D., van Leeuwaarden, J.S.H.: Multi-Access, Reservations and Queues. Springer, New York (2008)

8. Dieudonne, J.: Calcul Infinitésimal. Hermann, Paris (1980)

9. Drmota, M.: Systems of functional equations. Random Struct. Algorithms 10, 103-124 (1997)

10. Fayolle, G., Iasnogorodski, R.: Two coupled processors: The reduction to a Riemann-Hilbert problem. Z. Wahrscheinlichkeitstheor. Verw. Geb. 47, 325-351 (1979)

11. Fayolle, G., Iasnogorodski, R., Malyshev, V.: Random Walks in the Quarter Plane. Springer, New York (1999)

12. Flajolet, P., Sedgewick, R.: Analytic Combinatorics. Cambridge University Press, Cambridge (2009)

13. Flajolet, P., Gourdon, X., Martinez, C.: Patterns in random binary search trees. Random Struct. Algorithms 11, 223-244 (1988)

14. Flatto, L.: The longer queue model. Probab. Eng. Inf. Sci. 3, 537-559 (1989) 
15. Flatto, L., Hahn, S.: Two parallel queues created by arrivals with two demands. I. SIAM J. Appl. Math. 44, 1041-1053 (1984)

16. Foley, R.D., McDonald, D.R.: Join the shortest queue: Stability and exact asymptotics. Ann. Appl. Probab. 11, 569-607 (2001)

17. Foley, R.D., McDonald, D.R.: Large deviations of a modified Jackson network: Stability and rough asymptotics. Ann. Appl. Probab. 15, 519-541 (2005)

18. Foley, R.D., McDonald, D.R.: Bridges and networks: exact asymptotics. Ann. Appl. Probab. 15, 542586 (2005)

19. Guillemin, F., Pinchon, D.: Analysis of generalized processor-sharing systems with two classes of customers and exponential services. J. Appl. Probab. 41, 832-858 (2004)

20. Haque, L., Liu, L., Zhao, Y.Q.: Sufficient conditions for a geometric tail in a QBD process with countably many levels and phases. Stoch. Models 21, 77-99 (2005)

21. Ignatyuk, I.A., Malyshev, V.A., Scherbakov, V.V.: Boundary effects in a large deviation problem. Russ. Math. Surv. 49, 41-99 (1994)

22. Kroese, D.P., Scheinhardt, W.R.W., Taylor, P.G.: Spectral properties of the tandem Jackson network, seen as a quasi-birth-and-death process. Ann. Appl. Probab. 14, 2057-2089 (2004)

23. Li, H., Zhao, Y.Q.: A retrial queue with a constant retrial rate, server downs and impatient customers. Stoch. Models 21, 531-550 (2005)

24. Miyazawa, M.: Tail decay rates in double QBD processes and related reflected random walks. Math. Oper. Res. 34, 547-575 (2009)

25. Miyazawa, M., Zhao, Y.Q.: The stationary tail asymptotics in the GI/G/1-type queue with countably many background states. Adv. Appl. Probab. 36, 1231-1251 (2004)

26. Neuts, M.F.: Matrix-Geometric Solutions in Stochastic Models, an Algorithmic Approach. Johns Hopkins University Press, Baltimore (1981)

27. Pemantle, R., Wilson, M.C.: Twenty combinatorial examples of asymptotics derived from multivariate generating functions. SIAM Rev. 50, 199-272 (2008)

28. Resing, J.A.C., Örmeci, L.: A tandem queueing model with coupled processors. Oper. Res. Lett. 31, 383-389 (2003)

29. Sakuma, Y., Miyazawa, M.: On the effect of finite buffer truncation in a two-node Jackson network. J. Appl. Probab. 42, 199-222 (2005)

30. Shwartz, A., Weiss, A.: Large Deviations for Performance Analysis, Queues, Communications and Computing. Chapman \& Hall, London (1995)

31. Takahashi, Y., Fujimoto, K., Makimoto, N.: Geometric decay of the steady-state probabilities in a quasi-birth-and-death process with a countable number of phases. Stoch. Models 17, 1-24 (2001)

32. van Leeuwaarden, J.S.H., Resing, J.A.C.: A tandem queue with coupled processors: computational issues. Queueing Syst. 51, 29-52 (2005) 